\title{
Equilibrium gravity segregation in porous media with capillary heterogeneity
}

\author{
Avinoam Rabinovich ${ }^{1, \dagger}$ and Kan Bun Cheng ${ }^{1}$ \\ ${ }^{1}$ School of Mechanical Engineering, Tel-Aviv University, Tel-Aviv 69978, Israel
}

(Received 27 July 2019; revised 18 November 2019; accepted 11 February 2020)

We study the equilibrium of two phases following gravity segregation under the influence of capillary heterogeneity. Such processes are important in a number of porous media applications, e.g. determining reservoir composition, secondary migration, gravity drainage enhanced oil recovery and $\mathrm{CO}_{2}$ storage in aquifers. Solutions are derived for three-dimensional saturation distribution $S_{w}(x, y, z)$ and given as an analytical formula apart from a constant $P_{c}^{0}$ which is determined by numerical integration. The first solution assumes hydrostatic pressure and applies to cases without capillary entry pressure $\left(P_{c}\left(S_{w}=1\right)=0\right)$. The solution can be used for validation of numerical simulations and we show a close match for a number of cases. A second analytical solution is derived, extending the first, to cases of random log-normally distributed permeability fields. A formula for ensemble average saturation solution is presented and a comparison to solutions of various realizations is discussed. When capillary entry pressure is present, the solution based on hydrostatic pressure may be inaccurate due to entry pressure trapping which occurs when regions of $S_{w}=1$ are present. Using numerical simulation, we extend the solution to include estimations of entry pressure trapping for a range of parameters and show its applicability. The comparison of analytical and numerical results helps illustrate and draw insight on the trapping mechanism.

Key words: porous media

\section{Introduction}

Two-phase gravity segregation in porous media is a process by which phases separate into layers according to their density, with the heavier (e.g. liquid) phase at the bottom and lighter (e.g. gas) phase at the top. When steady-state is reached, the phases are in equilibrium, flow ceases and phases are perfectly layered. Capillary pressure effects substantially impact this process leading to saturation gradients, i.e. regions of mixed phases. Spatial variation of capillary pressure functions with permeability is often referred to as capillary heterogeneity and has an even more significant impact, leading to trapped, immobile regions in which the fluid and gas do not segregate at all. This process is important in a number of applications

$\dagger$ Email address for correspondence: avinoamr@tauex.tau.ac.il 
pertaining to various scales, from reservoir to laboratory, e.g. determining reservoir composition, secondary migration, gravity drainage enhanced oil recovery and $\mathrm{CO}_{2}$ storage in aquifers. In this work we derive an analytical solution to gravity-capillary equilibrium saturation distribution and apply it to investigate many different cases. Our main focus is comparing the analytical solution to dynamic numerical simulations for validation and to understand discrepancies between them. This allows us to determine the capabilities and limitations of both solutions as well as to obtain insight on capillary heterogeneity trapping.

A large body of existing literature deals with gravity segregation under the influence of capillary pressure effects. The first class of investigations aim at characterizing hydrocarbon reservoirs (Lee 1989; Wheaton 1991; Shapiro \& Stenby 1996; Montel et al. 2007; Bedrikovetsky 2013). These are closely related to this work since they consider no external pressure gradient (e.g. wells) or natural background flow and are interested in the equilibrium conditions of the phases under gravity and capillary forces. However, they focus on determining the composition of the phases taking into account thermal gradients, whereas our focus is on the impact of capillary heterogeneity. Additional relevant literature is related to gravity drainage enhanced oil recovery (e.g. Hagoort 1980; Donato, Tavassoli \& Blunt 2006), yet these investigations also tend to simplify the effects of capillarity.

Capillary heterogeneity effects are studied in a rich body of literature related to geologic carbon storage (Perrin \& Benson 2010; Krevor et al. 2011; Krause 2012; Wei et al. 2014; Kuo \& Benson 2015; Li \& Benson 2015; Pini \& Benson 2017). In this application, capillarity is responsible for trapping of $\mathrm{CO}_{2}$ within the surrounding aquifer brine and is considered to be of paramount importance for safe and long term sequestration (Hovorka et al. 2004; Han et al. 2010; Pentland et al. 2011; Deng et al. 2012; Krevor et al. 2012; Green \& Ennis-King 2013; Krevor et al. 2015). When considering the post-injection migration and trapping of $\mathrm{CO}_{2}$ it is often assumed that gravity and capillary forces dominate, as we assume in this work. Many numerical and analytical investigations have been published in effort to estimate the migration of $\mathrm{CO}_{2}$ following injection into an aquifer.

The first and most common type of study assumes that gravity segregation occurs rapidly and therefore the problem is reduced to a two-dimensional (or one-dimensional) lateral migration of the plume, occurring near the caprock at the aquifer top. Then the vertical equilibrium approximation can be employed, which has been implemented in numerical models and analytical solutions (often assuming a sharp interface) (Nordbotten \& Celia 2006; Dentz \& Tartakovsky 2009; Gasda, Nordbotten \& Celia 2009; MacMinn \& Juanes 2009; Juanes, MacMinn \& Szulczewski 2010; Loubens \& Ramakrishnan 2011; Nordbotten \& Dahle 2011; Gasda, Nilsen \& Dahle 2013; Andersen, Gasda \& Nilsen 2015; Nilsen, Lie \& Andersen 2016; Malekzadeh, Heidari \& Dusseault 2017). Similarly, gravity current models have been proposed for the migration of $\mathrm{CO}_{2}$ beneath a confining boundary (Hesse et al. 2007; Hesse, Orr \& Tchelepi 2008; Golding \& Huppert 2010; Golding et al. 2011).

A second type of study, more relevant to this work, focuses on the trapping that occurs during vertical migration of the $\mathrm{CO}_{2}$ plume as it rises towards the impermeable caprock (Bryant, Lakshminarasimhan \& Pope 2006; Juanes et al. 2006; Ide, Jessen \& Orr 2007; Hesse \& Woods 2010; Gershenzon et al. 2014; Li \& Benson 2015; Trevisan, Krishnamurthy \& Meckel 2017a). In particular, the literature related to upscaling $\mathrm{CO}_{2}$ storage migration employs these types of vertical migration flow simulations (Mouche, Hayek \& Mügler 2010; Saadatpoor, Bryant \& Sepehrnoori 2011; Behzadi \& Alvarado 2012; Rabinovich, Itthisawatpan \& Durlofsky 2015). Due 
to the complexity of solving three-dimensional dynamic $\mathrm{CO}_{2}$ migration problems (heterogeneity and time evolution must be incorporated to model trapping accurately) the models used previously were all numerical. Using numerical simulation for flow with capillary heterogeneity often encounters difficulties. The computational time becomes extremely long with increasing capillary effects and convergence is more difficult. Some ambiguities related to the solutions have also been reported (Rabinovich et al. 2015) and rigorous benchmarking has yet to be conducted. Furthermore, for stochastic analysis, numerical simulations must be used via a Monte Carlo approach, which is most likely not feasible due to the computational demand. Analytical solutions are therefore instrumental to $\mathrm{CO}_{2}$ vertical migration modelling and can also offer additional insight into the physical processes.

In this work, we derive an analytical solution to three-dimensional (3-D) saturation distribution in gravity segregation with capillary heterogeneity at equilibrium conditions. The solution assumes hydrostatic pressure variation and takes advantage of the capillary pressure - saturation constitutive relation $P_{c}\left(S_{w}\right)$ to derive a formula for the saturation spatial variations with permeability and porosity. While similar solutions have been derived in the past by Nordbotten \& Dahle (2010), Nordbotten \& Celia (2011) and Smith (2012), this work has a number of significant novelties. First, the solution is investigated considering three-dimensional problems with significant capillary heterogeneity. Comparisons with 3-D simulations are held to show how the solution can validate numerical codes and also to gain insight by analysing differences in the solutions. Second, an extension of the analytic solution is derived for random permeability fields. Finally, another extension is presented incorporating capillary entry pressure trapping in the solution, without the use of percolation considerations.

The solution is derived separately for two types of $P_{c}$ curves: with and without entry pressure. A wide variety of cases with conditions ranging from gravity dominated to capillary limit are studied by comparing the solution against numerical simulations. We show that for the case with capillary entry pressure a special and well known type of entry pressure trapping occurs which does not comply with the hydrostatic assumption and thus fails to be estimated by the analytical solution.

Capillary trapping of fluids is the result of pore scale mechanisms related to surface tension and snap-off phenomenon (Lenormand, Zarcone \& Sarr 1983; Pinder \& Gray 2008; Dullien 2012). However, we focus on the macroscale in which these processes are encapsulated in the $P_{c}$ function. We find that trapping in equilibrium conditions occurs due to capillary heterogeneity in two different configurations. The first is driven by a capillary pressure vertical gradient in combination with $P_{c}$ function spatial variations. The second is a result of entry pressure differences associated with regions which are fully wetting phase saturated. This entry pressure trapping is not captured by the analytical solution. Previous literature has addressed this phenomenon and invasion percolation methods have been developed to model the process (Ioannidis, Chatzis \& Dullien 1996; Carruthers \& van Wijngaarden 2000), mostly in numerical algorithms (Oldenburg, Mukhopadhyay \& Cihan 2016; Trevisan, Illangasekare \& Meckel 2017b) or for upscaling purposes (Yortsos et al. 1993; Nooruddin \& Blunt 2018). We take a different approach here, modifying the analytical solution using a heuristic method based on matching simulation trapping results. For a range of parameter values, the new solution is shown to estimate the overall trapped $\mathrm{CO}_{2}$ adequately.

One of the major challenges in flow modelling of subsurface formations is the uncertainty associated with the porous rock properties. Permeability $(k)$ is usually measured in a small number of locations while it typically varies by orders of 
magnitude over small length scales. It is therefore common to model $k$ as random and seek the expected value of the flow solution (Haldorsen, Brand \& Macdonald 1987; Dongxiao \& Tchelepi 1999; Rabinovich, Dagan \& Miloh 2012; Cheng, Rabinovich \& Dagan 2019). In this work we extend the problem for the case without entry pressure effects to include log-normally distributed random $k$. An analytical solution to the expected value of saturation is obtained by ensemble averaging and the result is validated by comparison to solutions of many realizations.

The paper is organized as follows. In $\S 2$ we detail the problem and relevant equations. Section 3 presents derivation of the analytical solution including an ensemble mean solution for the case of random $k$. Section 4 presents results for cases without entry pressure trapping (van Genuchten type $P_{c}$ ). Section 5 discusses results for Brooks-Corey $P_{c}$, which incorporates entry pressure trapping, and presents a method for estimating the trapped $\mathrm{CO}_{2}$. The summary and conclusions of this work are given in $\S 6$.

\section{Problem statement}

We consider a porous medium surrounded by impermeable, no-flow boundaries. This could represent any type of closed reservoir in a field study or sealed rock sample in a laboratory experiment. The medium contains two phases - wetting $(w)$, e.g. a liquid and non-wetting $(n w)$, e.g. a gas, with different densities $\rho_{w}$ and $\rho_{n w}$. The phases are distributed throughout the domain with some initial saturation $S_{w}^{\text {init }}(x, y, z)$, where $S_{w}$ is wetting phase saturation, $S_{w}+S_{n w}=1$ and a Cartesian system is used for spatial coordinates. The phases will migrate due to buoyancy and capillary forces until a steady-state is reached, when the fluid and gas are in equilibrium. We seek the saturation distribution $S_{w}(x, y, z)$ at equilibrium, i.e. the final location of the phases.

The equations for the general problem described above are considered to be those describing immiscible flow of two incompressible phases in an incompressible rock and given by mass conservation

$$
\phi \frac{\partial S_{j}}{\partial t}+\nabla \cdot \boldsymbol{u}_{j}=0,
$$

and Darcy's law

$$
\boldsymbol{u}_{j}=-\frac{k_{r j}}{\mu_{j}} \boldsymbol{k} \cdot \nabla\left(p_{j}+\rho_{j} g z\right),
$$

where $\phi$ is the porosity of the rock, $k_{r j}$ the relative permeability to phase $j$ ( $j=w$ or $j=n w), \mu_{j}$ the viscosity of phase $j, p_{j}$ the pressure of phase $j, \boldsymbol{u}_{j}$ the Darcy velocity of phase $j, \boldsymbol{k}$ the absolute permeability tensor, $g$ is gravitational acceleration and $z$ is the vertical coordinate. Pressures of the non-wetting phase and the wetting phase are related by

$$
p_{n w}-p_{w}=P_{c}\left(S_{w}\right),
$$

where $P_{c}\left(S_{w}\right)$ is the capillary pressure curve.

When considering gravity driven flow, as opposed to flow driven by injection or production wells, capillary pressure is usually significant and different structures of $P_{c}\left(S_{w}\right)$ curves will have a drastic effect on the solution. While some gravity segregation models assume the same curves throughout the domain, it is generally more accurate to take into account the spatial change of $P_{c}\left(S_{w}\right)$. This is typically modelled using the Leverett $J$-function as follows:

$$
P_{c}\left(S_{w}, k, \phi\right)=\alpha C \sqrt{\frac{\phi}{k}} J\left(S_{w}\right),
$$


where $C$ is a fitting parameter and $\alpha$ is the $J$-function scaling coefficient, which accounts for interfacial tension, contact angle and unit conversion. Isotropic permeability is assumed here so that $k$ is a scalar function. This is not a requirement for the derivation, but assumed for simplicity to avoid defining $P_{c}$ as a function of the directional components of $k$ (often taken as the average). Two of the most widely used $J$-functions are the van Genuchten (VG) (van Genuchten 1980) \& Brooks-Corey (BC) (Brooks \& Corey 1966) models given by

$$
J_{V G}\left(S_{w}\right)=\left[\left(\widetilde{S}_{w}\right)^{-1 / m}-1\right]^{1-m}
$$

and

$$
J_{B C}\left(S_{w}\right)=\left(\widetilde{S}_{w}\right)^{-1 / \lambda},
$$

respectively, where $\widetilde{S}_{w}=\left(S_{w}-S_{w i}\right) /\left(1-S_{w i}\right), S_{w i}$ is the irreducible water saturation and $\lambda, m$ are fitting parameters. It is evident that hysteresis is not considered in our capillary pressure models (Hilfer 2006; Pini \& Benson 2017). Furthermore, the BC model incorporates capillary entry pressure, i.e. $P_{c}\left(S_{w}=1\right) \neq 0$ while in the VG model entry pressure is zero.

We are interested in the solution after the phases have equilibrated and there is no longer any flow. Under these conditions phase velocities are zero everywhere, i.e. $\boldsymbol{u}_{j}=$ 0 , and we immediately obtain from (2.2) that

$$
\begin{gathered}
p_{w}+\rho_{w} g z=\text { const. } \\
p_{n w}+\rho_{n w} g z=\text { const. }
\end{gathered}
$$

which is the hydrostatic phase pressure variation. Subtracting $(2.7 a)$ from $(2.7 b)$ leads to an expression for the capillary pressure distribution

$$
P_{c}=P_{c}^{0}+\Delta \rho g z
$$

where $\Delta \rho=\rho_{w}-\rho_{n w}$ and $P_{c}^{0}$ (the capillary pressure at $z=0$ ) is a constant to be determined. Next, we integrate (2.1) over the entire domain and apply the divergence theorem to arrive at the equation

$$
\int_{V} \widetilde{S}_{w}(x, y, z) \mathrm{d} V=\int_{V} \widetilde{S}_{w}^{\text {init }} \mathrm{d} V,
$$

where the integration here is a volume integral (i.e. triple integral) over the domain volume $V$ and $\widetilde{S}_{w}^{\text {init }}=\left(S_{w}^{\text {init }}-S_{w i}\right) /\left(1-S_{w i}\right)$. Equation (2.9) is simply a statement that total saturation in the domain remains constant over time since we consider a closed reservoir with no flow through the boundaries. It can be expressed in terms of average saturation, i.e. $\left\langle\widetilde{S}_{w}\right\rangle=\left\langle\widetilde{S}_{w}^{\text {init }}\right\rangle$, where \langle\rangle denotes averaging over the entire domain.

The final equation for our problem is the capillary pressure-saturation relationship which can be generally expressed as $P_{c}=f\left(\phi, k, S_{w}\right)$, where $f$ is some known function obtained through laboratory experiments on rock samples. In this work, $f$ is given by (2.4) and (2.5) or (2.6). Equations (2.8), (2.9) and (2.4) are a system of three equations which can be solved to obtain $P_{c}^{0}, P_{c}(z)$ and $S_{w}(x, y, z)$. In general, the saturation solution will be obtained by inverting (2.4) and will depend on spatial variation of porosity and permeability as well as the hydrostatic capillary pressure.

We now formulate the governing equations in dimensionless form by defining the following non-dimensional parameters: $N_{b}=\alpha C /\left(\Delta \rho g h^{2}\right), \widetilde{k}=k / h^{2}, \widetilde{P}_{c}^{0}=P_{c}^{0} /(\Delta \rho g h)$, 
$\widetilde{P}_{c}=P_{c} /(\Delta \rho g h)$ and $\widetilde{z}=z / h$, where $h$ is the domain height and $N_{b}$ is the Bond number representing a ratio between capillary and gravity forces. Substituting these in the governing equations (2.8) and (2.4) we arrive at

$$
\widetilde{P}_{c}=\widetilde{P}_{c}^{0}+\widetilde{z}
$$

and

$$
\widetilde{P}_{c}=N_{b} \sqrt{\frac{\phi}{\widetilde{k}}} J\left(S_{w}\right) .
$$

The third governing equation is already dimensionless and remains in the form presented in (2.9).

\subsection{Problem assumptions}

All the assumptions used in the formulation and in the next section for deriving the solution are detailed as follows: (i) flow obeying Darcy's law, (ii) immiscible fluids, (iii) incompressible medium and fluids, (iv) isotropic permeability, (v) Leverett $J$-function scaling of the capillary pressure, (vi) $J$-function of type VG or BC and (vii) equilibrium conditions.

\section{Analytical solution}

We now derive a solution to the problem formulated in the previous section. It is important to point out that the solution described by (2.9)-(2.11) does not fully account for entry-pressure trapping of phases. When entry pressure is introduced in (2.6), regions with non-zero capillary pressure can be fully saturated with water. This occurs when $\widetilde{P}_{c}<\widetilde{P}_{e}$, where $\widetilde{P}_{e}=N_{b} \sqrt{\phi / \widetilde{k}}$ is the entry pressure. The non-wetting phase will invade these regions only if the surrounding capillary pressure exceeds $\widetilde{P}_{e}$. In these regions, equation (2.10) does not hold and yet the condition $\boldsymbol{u}_{j}=0$ is still fulfilled. We will refer to this type of trapping involving fully saturated regions as entry pressure trapping. In the following, we continue to derive a solution without considering entry pressure trapping, which will be discussed in detail in $\S 5$.

Taking capillary pressure described by (2.11) together with a $J$-function of the form in (2.5) or (2.6), leads to $\widetilde{P}_{c}>0$. However, equation (2.10) is not compatible with this requirement and may result in negative $\widetilde{P}_{c}$ values. We therefore modify (2.10) to avoid negative capillary pressure

$$
\widetilde{P}_{c}= \begin{cases}\widetilde{P}_{c}^{0}+\widetilde{z}, & \widetilde{z}>-\widetilde{P}_{c}^{0} \\ 0, & \widetilde{z} \leqslant-\widetilde{P}_{c}^{0} .\end{cases}
$$

We note that we have considered here $\Delta \rho>0$ (heavier wetting phase) by convention, however, this equation can apply to both cases of $\Delta \rho>0$ and $\Delta \rho<0$ (heavier nonwetting phase). In the first case, $z=0$ is at the top of the domain so that $P_{c}$ decreases with depth, while for the latter case, $z=0$ is at the bottom of the domain and $P_{c}$ increases with depth.

We can now obtain a formula for the saturation distribution by substituting (2.11) with the $J$-function from (2.6) or (2.5) in (3.1) and solving for $\widetilde{S}_{w}$. This results in the 
solution

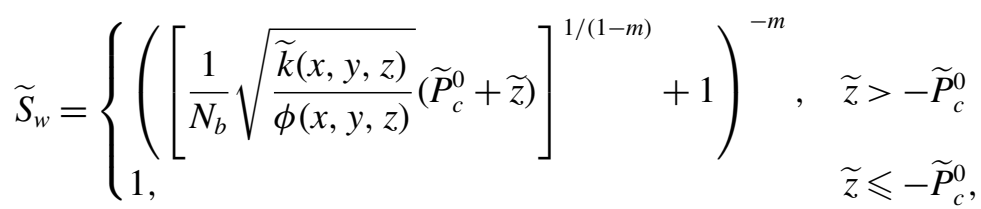

for the VG $J$-function and

$$
\widetilde{S}_{w}= \begin{cases}{\left[\frac{1}{N_{b}} \sqrt{\frac{\widetilde{k}(x, y, z)}{\phi(x, y, z)}}\left(\widetilde{P}_{c}^{0}+\widetilde{z}\right)\right]^{-\lambda},} & \widetilde{z}>\widetilde{P}_{e}-\widetilde{P}_{c}^{0} \\ 1, & \widetilde{z} \leqslant \widetilde{P}_{e}-\widetilde{P}_{c}^{0},\end{cases}
$$

for the BC $J$-function. Despite the exclusion of capillary entry trapping in the solution, there is still an impact of $\widetilde{P}_{e}$, which can be seen in (3.3), where saturation is 1 if $\widetilde{P}_{c} \leqslant \widetilde{P}_{e}$.

The constant $\widetilde{P}_{c}^{0}$ is obtained by substituting (3.2) or (3.3) in (2.9) and solving the integral equation. For some special cases the integral can be solved analytically. This is the case for a homogeneous medium $(k=$ const. and $\phi=$ const.) with $\mathrm{BC}$ type capillary pressure, which leads to the equations

$$
\begin{gathered}
\left(\widetilde{P}_{c}^{0}\right)^{1-\lambda}-\left(1+\widetilde{P}_{c}^{0}\right)^{1-\lambda}=(\lambda-1) \widetilde{P}_{e}^{\lambda} \int_{V} \widetilde{S}_{w}^{\text {init }} \mathrm{d} V, \text { if } \widetilde{P}_{e}-\widetilde{P}_{c}^{0} \geqslant 1, \\
\widetilde{P}_{c}^{0}-\frac{\widetilde{P}_{e}^{\lambda}\left(\widetilde{P}_{c}^{0}\right)^{1-\lambda}}{1-\lambda}=\frac{\lambda \widetilde{P}_{e}}{\lambda-1}-1+\int_{V} \widetilde{S}_{w}^{\text {init }} \mathrm{d} V \quad \text { if } \widetilde{P}_{e}-\widetilde{P}_{c}^{0}<1 .
\end{gathered}
$$

However, for most cases with general functions $\widetilde{k}(x, y, z)$ and $\phi(x, y, z)$, equation (2.9) can be solved numerically to obtain $\widetilde{P}_{c}^{0}$, particularly when considering discrete, noncontinuous, permeability and porosity distributions. This is the approach we take to obtain the results presented in this work.

\subsection{Ensemble mean solution}

Many applications of flow modelling in porous rocks must incorporate permeability uncertainty, i.e. $k$ values are generally unknown and vary by orders of magnitude over small distances. A common approach for dealing with this problem is to consider $k$ as a random space function (RSF) with known statistical properties and to seek the ensemble mean of the variable of interest. We now extend the previous formulation by considering $k$ to be a stationary and isotropic RSF, log-normally distributed so that the $Y=\ln k$ (log permeability) is characterized by the mean $\langle Y\rangle=\ln k_{G}$ and variance $\sigma_{y}^{2}$. The probability density function of $Y$ is then given by

$$
F(Y)=\frac{1}{\sqrt{2 \pi \sigma_{y}^{2}}} \exp \left[\frac{-\left(Y-\ln k_{G}\right)^{2}}{2 \sigma_{y}^{2}}\right] \text {. }
$$

The ensemble average over many realizations of permeability $k$ can be calculated by the following expression:

$$
\left\langle\widetilde{S}_{w}\right\rangle_{E}=\int_{-\infty}^{\infty} \widetilde{S}_{w}(\widetilde{y}, \widetilde{z}) \cdot F(\widetilde{y}) \mathrm{d} \widetilde{y},
$$

where \langle\rangle$_{E}$ denotes ensemble average (or expected value) and $\widetilde{y}=\ln \widetilde{k}$. 
First, we consider the case of VG capillary pressure. Substituting (3.2) in equation, (3.6) we arrive at

$$
\left\langle\widetilde{S}_{w}\right\rangle_{E}= \begin{cases}\int_{-\infty}^{\infty}\left[A\left(\widetilde{P}_{c}^{0}, \widetilde{z}\right) \exp \left[\frac{\widetilde{y}}{2(1-m)}\right]+1\right]^{-m} F(\widetilde{y}) \mathrm{d} \widetilde{y}, & \widetilde{z}>-\widetilde{P}_{c}^{0} \\ 1, & \widetilde{z} \leqslant-\widetilde{P}_{c}^{0},\end{cases}
$$

where $A=\left[\left(\widetilde{P}_{c}^{0}+\widetilde{z}\right) /\left(N_{b} \sqrt{\phi}\right)\right]^{1 /(1-m)}$ includes all the deterministic parameters. Porosity is considered here to be constant as it typically varies much less than $k$. The integral in (3.7) can be solved numerically, however, we continue the derivation to arrive at a fully analytical expression. The details are presented in appendix A and the final result is given by

$$
\left\langle\widetilde{S}_{w}\right\rangle_{E}= \begin{cases}\sum_{n=0}^{\infty}\left(\begin{array}{c}
-m \\
n
\end{array}\right)\left\{A^{n} B(n) \operatorname{erfc}[C(n)],\right. & \widetilde{z}>-\widetilde{P}_{c}^{0} \\
\left.+A^{-n-m} B(-n-m) \operatorname{erfc}[-C(-n-m)]\right\}, & \widetilde{z} \leqslant-\widetilde{P}_{c}^{0},\end{cases}
$$

where

$$
\begin{gathered}
B(\chi)=\frac{1}{2} \exp \left\{\chi\left[\chi \sigma_{y}^{2}-4(m-1) \ln \widetilde{k}_{G}\right] /\left[8(m-1)^{2}\right]\right\}, \\
C(\chi)=-\frac{\chi \sigma_{y}^{2}+2(m-1)\left[2(m-1) \ln A-\ln \widetilde{k}_{G}\right]}{\sqrt{8 \sigma_{y}^{2}} \cdot(m-1)},
\end{gathered}
$$

and $\left(\begin{array}{c}-m \\ n\end{array}\right)=-m \cdot(-m-1) \cdot \ldots(-m-n+1) / n !$ are the binomial coefficients for any real valued $m$ and natural number $n$. The solution for mean saturation is thus given by (3.8) together with (2.9) which is used for obtaining $\widetilde{P}_{c}^{0}$ in $A$.

Next, we consider BC capillary pressure and substitute equation (3.3) into (3.6). The piecewise function in this case depends on the variable of integration $\widetilde{y}$ since $\widetilde{P}_{e}$ is a function of permeability. Therefore we separate the integration limits into two ranges as follows:

$$
\left\langle\widetilde{S}_{w}\right\rangle_{E}=\left(\frac{\widetilde{P}_{c}^{0}+\widetilde{z}}{N_{b} \sqrt{\phi}}\right)^{-\lambda} \int_{-\infty}^{\widetilde{y}^{*}} \exp [-\lambda \widetilde{y} / 2] F(\widetilde{y}) \mathrm{d} \widetilde{y}+\int_{\widetilde{y}^{*}}^{\infty} F(\widetilde{y}) \mathrm{d} \widetilde{y},
$$

where $\widetilde{y}^{*}=-\lambda \ln D$ is the value of $\widetilde{y}$ in which $\widetilde{P}_{e}=\widetilde{P}_{c}^{0}$ and $D=\left(\widetilde{P}_{c}^{0}+\widetilde{z}\right) /\left(N_{b} \sqrt{\phi}\right)$. Solving the integrals in (3.9) and rearranging we arrive at the solution

$$
\begin{aligned}
\left\langle\widetilde{S}_{w}\right\rangle_{E}= & \frac{1}{2} D^{-\lambda} \exp \left[\lambda\left(\frac{\lambda}{8} \sigma_{y}^{2}-4 \ln \widetilde{k}_{G}\right)\right] \operatorname{erfc}\left[\frac{-2 \ln \widetilde{k}_{G}-\lambda \ln D+\lambda \sigma_{y}^{2}}{\sqrt{8 \sigma_{y}^{2}}}\right] \\
& +\frac{1}{2} \operatorname{erfc}\left[\frac{\lambda \ln D+\ln \widetilde{k}_{G}}{\sqrt{2 \sigma_{y}^{2}}}\right] .
\end{aligned}
$$

The above solution does not incorporate entry-pressure trapping, as mentioned previously regarding the formulation of (3.3). 


\section{Results - no entry pressure}

This section will present an analysis of the solution derived previously considering cases without capillary entry pressure. Capillary pressure is modelled with the VG relationship given by (2.4) with heterogeneous permeability $k(x, y, z)$ and (2.5) as the $J$-function. Throughout this work, analytical solutions will be compared to numerical results obtained using Stanford's General Purpose Research Simulator, (known as GPRS) (Cao 2002) on simulation grids of $25 \times 25 \times 50$. A finer grid of $50 \times 50 \times 100$ was also used to test for convergence, which was in fact achieved. Furthermore, in the following, the non-wetting phase will be addressed as $\mathrm{CO}_{2}$ and the wetting phase as water, keeping in mind $\mathrm{CO}_{2}$ storage applications.

A permeability realization is generated using the sequential Gaussian simulation (Deutsch \& Journel 1992) module of the Stanford Geostatistical Modeling Software, (known as SGeMS) (Remy, Boucher \& Wu 2009). The realization is taken to have geometric mean $k_{G}=100 \mathrm{md}$, variance of $\log$ permeability $\sigma_{y}^{2}=1(Y=\ln k)$ and dimensionless correlation length $l_{x}=l_{y}=l_{z}=0.1$ in the $x, y$ and $z$ directions (non-dimensionalized by the domain length in the corresponding direction). The dimensionless permeability is then substituted in (3.2) and together with the solution to (2.9) for $\widetilde{P}_{c}^{0}$, we obtain the saturation distribution. Porosity is taken to be constant $(\phi=0.25)$ for simplicity, since it usually has much smaller variations than permeability. Values of $\Delta \rho g=9.16 \mathrm{kPa} \mathrm{m}^{-1}, m=0.75$ and $h=0.098 \mathrm{~m}$ are assumed and used also for simulation input.

Figure 1 presents results for $\mathrm{CO}_{2}$ saturation distribution in a slice through the centre of the cubical domain for three different Bond numbers: a small value of $N_{b}=7.8$, a medium value of $N_{b}=78$ and a large value of $N_{b}=780$. Parameter values in this work often seem arbitrary because they are generally calculated from dimensional values which are used as input in the simulator. The left-hand column of plots $(a, c, e)$ are analytical results while the right-hand column $(b, d, f)$ are simulation results. A uniform initial mixture of water and $\mathrm{CO}_{2}$ is assumed in the domain, i.e. $\widetilde{S}_{w}^{\text {init }}=0.5$ is used in the simulations leading to $\left\langle\widetilde{S}_{w}^{\text {init }}\right\rangle=0.5$. It is clear that there is an excellent agreement between analytical and numerical solutions for all cases.

Saturation errors $(E)$ are presented in table 1 ('uniform initial'), calculated by $E=\left|S_{w}^{\text {numerical }}-S_{w}^{\text {analytical }}\right|$ at every grid block. Mean error is calculated as $\langle E\rangle$, averaging over all grid block errors. The portion of the domain with an error above a threshold value is calculated by $E_{a}=N(E>a) / N$, i.e. the number of grid blocks with error above threshold $a$ divided by the total number of grid blocks $(N)$. The results presented in table 1 show small errors in all cases and for all measure types. The above analysis is an example of utilizing the analytical solution for validation of a numerical code. In this specific case the match is excellent, showing that the simulation resolution is sufficiently high.

The case of small capillary pressure values corresponding to $N_{b}=7.8$ (figure $1 a, b$ ) shows a gravity dominated regime with almost complete segregation. Still, capillary pressure is not completely negligible and some spatial variation of $\widetilde{S}_{\mathrm{CO}_{2}}$ is seen as a result of trapping. The case of large capillary pressure corresponding to $N_{b}=780$ (figure $1 e, f$ ) shows a capillary dominated regime in which $\widetilde{S}_{\mathrm{CO}_{2}}$ is distributed throughout the domain with many regions of trapped phases. Increasing values of $N_{b}$ above 780 or decreasing below 7.8 will not have a significant impact on the solutions in figures $1(a, b)$ and $1(e, f)$, respectively. The case of $N_{b}=78$ in figure $1(c, d)$ shows a gravity-capillary regime in which both partial segregation and capillary effects are present. 
(a)

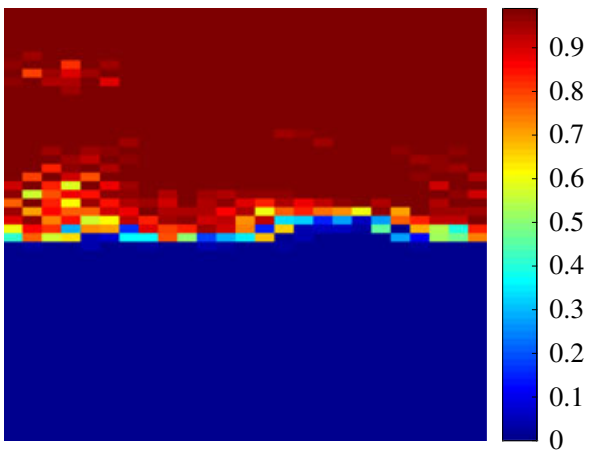

(c)

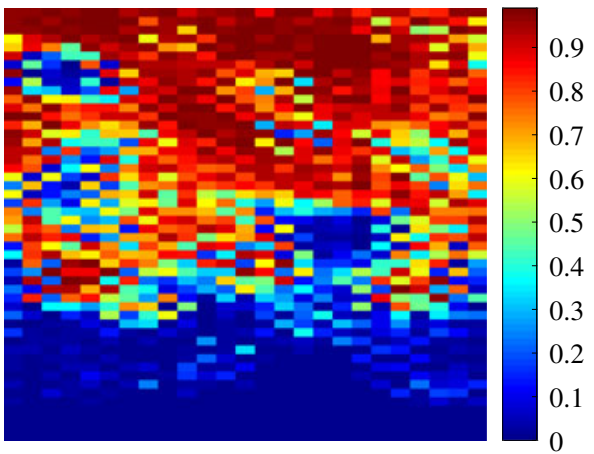

(e)



(b)

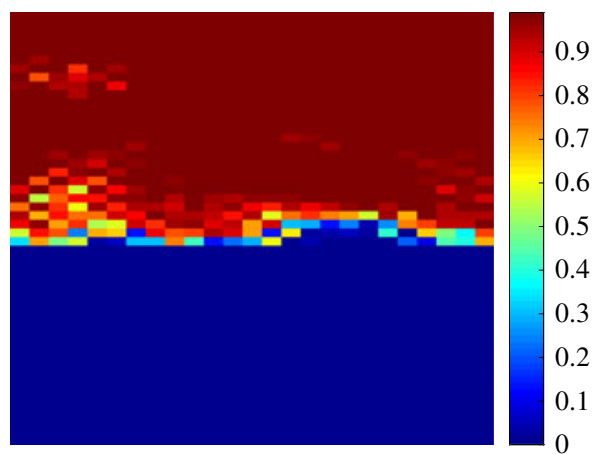

(d)

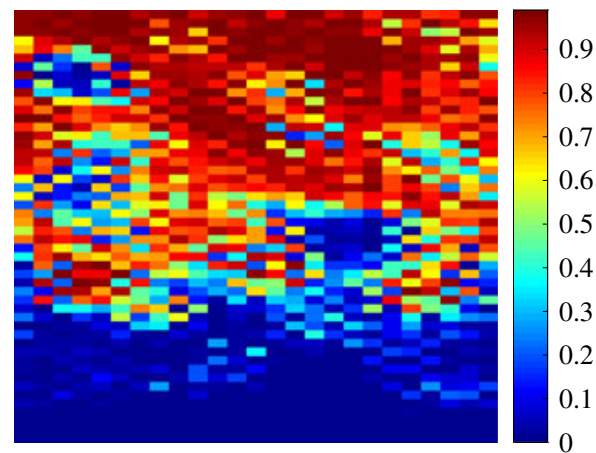

$(f)$

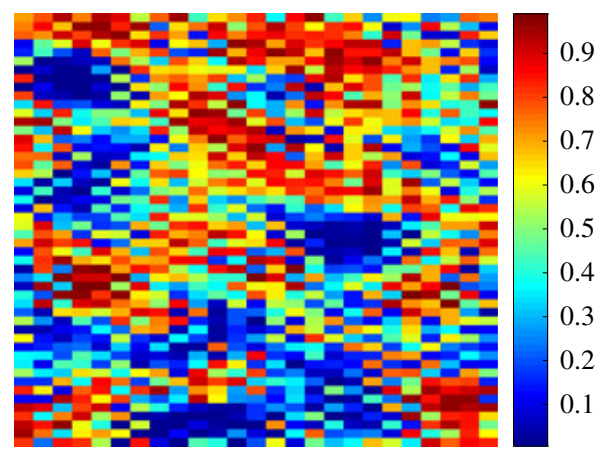

FIgURE 1. The $\mathrm{CO}_{2}$ saturation distribution in a vertical slice through the domain centre. Plots $(a, c, e)$ are results for analytical solution while $(b, d, f)$ are numerical simulations. The first row of plots $(\mathrm{a}, \mathrm{b})$ are results for $N_{b}=7.8$, the second row $(c, d)$ is for $N_{b}=78$ and the last row $(e, f)$ is for $N_{b}=780$.

Figure 2 presents plane averaged $\widetilde{S}_{w}$ as a function of $\widetilde{z}$. The transition from gravity dominated to capillary dominated conditions can be observed in the profiles. For $N_{b}=$ $7.8, \widetilde{P}_{c}$ values are small and gravity dominates, which results in significant segregation between phases (red curve) and many values of $\widetilde{S}_{w}=0$ or 1 in (3.2) (seen when taking $N_{b} \rightarrow 0$ ). For $N_{b}=780$, the $\widetilde{P}_{c}$ curve is large and therefore capillarity dominates, leading to capillary limit saturation distribution (light blue line), i.e. variation of $\widetilde{S}_{w}$ with $\widetilde{z}$ around the value of $\left\langle\widetilde{S}_{w}^{\text {init }}\right\rangle$. The variations are a results of heterogeneity $\widetilde{k}(x, y, z)$ and for a homogeneous medium the result would be simply $\widetilde{S}_{w}=\left\langle\widetilde{S}_{w}^{\text {init }}\right\rangle$. In between the 


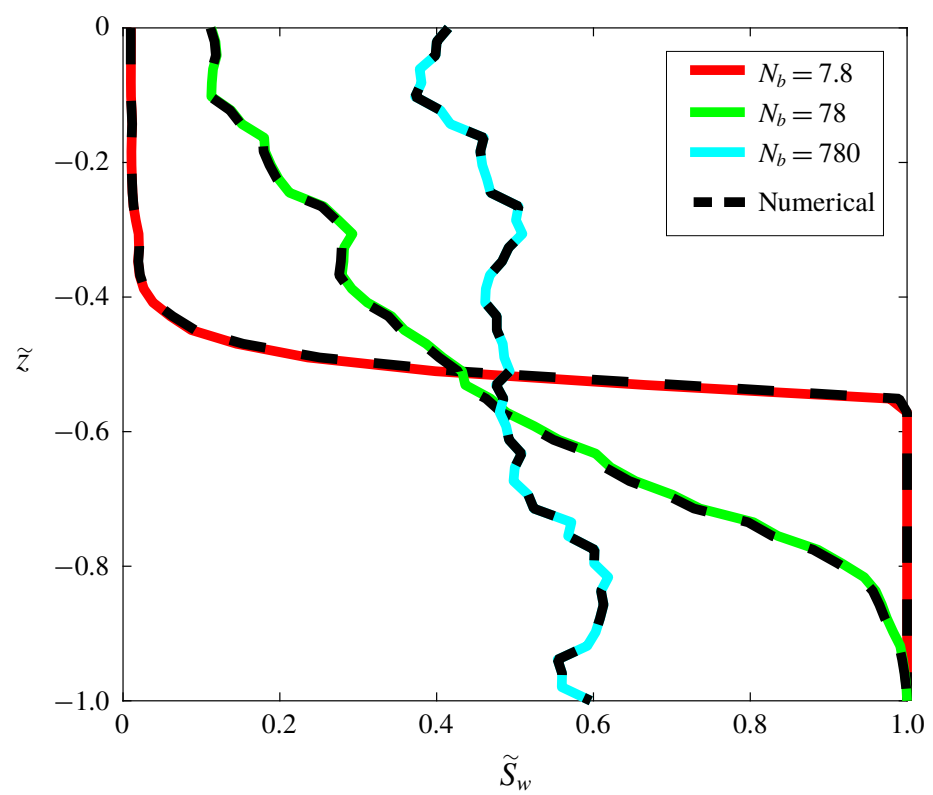

FIGURE 2. Horizontally averaged saturation as a function of height for different values of parameter $N_{b}$ considering VG capillary pressure. Analytical results (solid lines) given by (3.2) and (2.9) are compared with numerical simulation results (dashed lines).

\begin{tabular}{lccccccc}
\hline & \multicolumn{3}{c}{ Uniform initial } & & \multicolumn{3}{c}{ Bottom injection initial } \\
\cline { 2 - 4 } & $N_{b}=7.8$ & $N_{b}=78$ & $N_{b}=780$ & & $N_{b}=7.8$ & $N_{b}=78$ & $N_{b}=780$ \\
Mean error $(\langle E\rangle)$ & 0.0024 & 0.0036 & $1.4 \times 10^{-5}$ & & $5.2 \times 10^{-4}$ & $8.7 \times 10^{-4}$ & $5.3 \times 10^{-4}$ \\
Error above $0.1\left(E_{0.1}\right)$ & $0.03 \%$ & $0 \%$ & $0 \%$ & & $0 \%$ & $0 \%$ & $0 \%$ \\
Error above $0.05\left(E_{0.05}\right)$ & $1 \%$ & $0.003 \%$ & $0 \%$ & & $0.003 \%$ & $0 \%$ & $0 \%$ \\
TABLE 1. Error $E=\left|S_{w}^{\text {numerical }}(x, y, z)-S_{w}^{\text {analytical }}(x, y, z)\right|$ & for different values of $N_{b}$ and \\
considering two cases of initial conditions: uniform & $\widetilde{S}_{w}^{\text {init }}=0.5$ and bottom injection of \\
$\left\langle\widetilde{S}_{\mathrm{CO}_{2}}^{\text {init }}\right\rangle=0.1$ (figure $\left.3 a\right)$.
\end{tabular}

two limits of gravity and capillary dominance, e.g. $N_{b}=78$ in figure 2 , both gravity and capillary effects are apparent.

Figure 3(a-c) presents results for a different initial saturation distribution, i.e. not a constant $\widetilde{S}_{w}^{\text {init }}=0.5$ as previously considered. In general, the solution does not depend on the initial distribution and only requires knowledge of the total initial saturation (right-hand side of (2.9)). However, the numerical solution is obtained by simulating the full time evolution from initial condition to steady-state and therefore may be affected by $\widetilde{S}_{\mathrm{CO}_{2}}^{\text {init }}$ (due to numerical error). To test this, we assume an arbitrary saturation distribution shown in figure $3(a)$, obtained by injecting $\mathrm{CO}_{2}$ at the bottom of the domain. The steady-state equilibrium results are shown in figures $3(b)$ and $3(c)$ for analytical and numerical results, respectively. Excellent agreement can be seen. Further validation was conducted by a grid-by-grid error calculation and presented in table 1 ('bottom injection initial') where minuscule errors are shown. It is therefore 
(a)

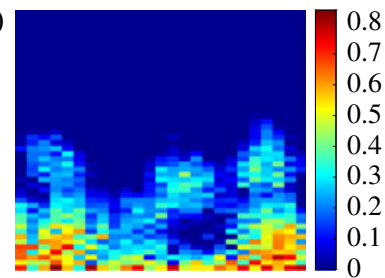

(b)

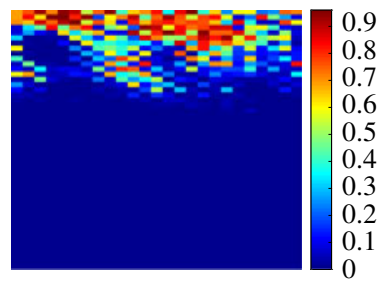

(c)

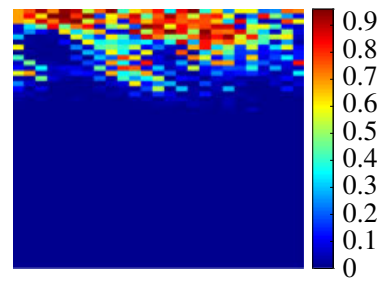

FIgURE 3. The $\mathrm{CO}_{2}$ saturation distribution in a vertical slice through the domain centre for $(a)$ initial time $\left(\widetilde{S}_{\mathrm{CO}_{2}}^{\text {init }}(x, y, z)\right),(b)$ equilibrium analytical solution and $(c)$ equilibrium numerical solution.

concluded that for the VG case, the numerical solution does not depend on the initial distribution, as expected. There is, however, a dependence on the overall average initial saturation $\left\langle\widetilde{S}_{\mathrm{CO}_{2}}^{\text {init }}\right\rangle$. The results in figure $3(b, c)$ are for the same parameter as in figure $1(c, d)$ and thus we can observe the impact of a smaller $\left\langle\widetilde{S}_{\mathrm{CO}_{2}}^{\text {init }}\right\rangle$. For the former case, $\left\langle\widetilde{S}_{\mathrm{CO}_{2}}^{\text {init }}\right\rangle=0.5$ while for the latter case $\left\langle\widetilde{S}_{\mathrm{CO}_{2}}^{\text {init }}\right\rangle=0.1$ and in fact the smaller $\mathrm{CO}_{2}$ region is apparent in figures $3(b)$ and $3(c)$.

\subsection{Ensemble mean results}

The ensemble average solution presented in $\$ 3.1$ will be discussed now. Figures 4 and 5 present ensemble mean saturation profiles given by (3.8) and (2.9). In figure $4(a)$ we present the case of $N_{b}=7.8, \phi=0.25, m=0.75,\left\langle\widetilde{S}_{\mathrm{CO}_{2}}^{\text {init }}\right\rangle=0.5$, $\sigma_{y}^{2}=4$ and $\ln \widetilde{k}_{G}=-25.3$ (corresponding to $k_{G}=100 \mathrm{md}$ ) using a dashed red line. We also plot horizontally averaged $S_{w}$ for the same problem parameters using 30 different $k$ realizations via calculations from (3.2) and (2.9) (thin grey lines). It is clear that the saturation profiles of the 30 realizations surround the ensemble mean solution closely and the scatter is quite small. This indicates that our derivation for $\left\langle\widetilde{S}_{w}\right\rangle_{E}$ is correct and that the $k$ sample size is sufficiently large so horizontal averaging (used to obtain the profiles) leads to fairly similar curves. Furthermore, we plot the ensemble average of the 30 realizations using a solid green line and show that the result coincides with the analytical ensemble mean. In fact only a few realizations are needed to converge to the green line.

Figure $4(b)$ shows that even the solution for a single realization (solid lines) follows the ensemble mean profiles (dashed lines) closely for three different cases with varying parameters. For sufficiently large $k$ samples, corresponding to a fine grid mesh, the horizontal averaging is responsible for the fairly close match. It is an expression of ergodic theory, stating that under conditions of $k$ stationarity and small correlation lengths compared with domain lengths $\left(l_{x}, l_{y}, l_{z} \ll 1\right)$, ensemble averaging can be substituted with spatial averaging. Figure $4(c)$ presents results for statistically anisotropic permeability. It is seen that heterogeneity structures which are horizontally elongated $\left(l_{x}, l_{y}>l_{z}\right)$ lead to significantly more erratic saturation curves as permeability differences between layers are increased and the condition $l_{x}, l_{y} \ll 1$ is fulfilled to a lesser extent. Nevertheless, the average saturation given by (3.8) and (2.9) does not depend on anisotropy and despite the large saturation variations between different layers, the ensemble average of the realizations (green dashed curve) matches the analytical result (red dashed curve). 

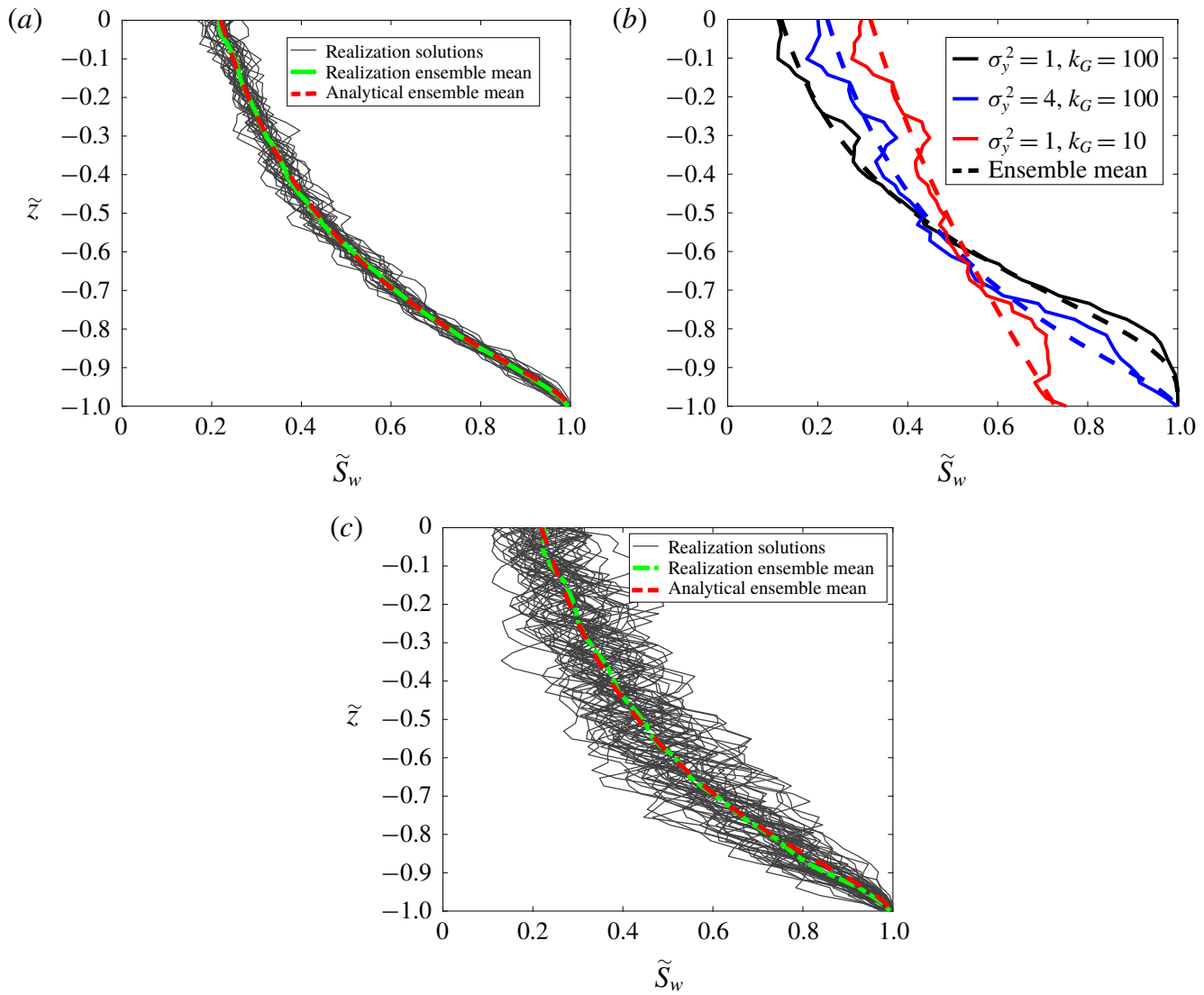

FIGURE 4. Comparison between ensemble mean solution (equation (3.8)) and solutions using permeability realizations (equation (3.2)) for (a) 30 realizations of the case $\sigma_{y}^{2}=4$, $\ln \widetilde{k}_{G}=-25.3,(b)$ one realization of three cases specified in legend and (c) 30 realizations for $\sigma_{y}^{2}=4, \ln \widetilde{k}_{G}=-25.3$ with $l_{x}=l_{y}=0.25$ and $l_{z}=0.1$.

The analytical solution given by (3.8) allows us to analyse the impact of heterogeneity on the saturation either by scanning different parameter values or by considering the limits of large/small parameters directly in the equations. Figure 5 illustrates the impact of log permeability variance on the solution. For low variance (e.g. $\sigma_{y}^{2}=0.01$ ) the solution tends to that of a homogeneous medium with permeability $k=k_{G}$, seen by comparing the black solid and yellow dashed curves. As $\sigma_{y}^{2}$ increases, there are more capillary effects leading to less variability of saturation and a smaller fully water saturated zone at the bottom of the domain. However, at the limit of high variance (e.g. $\left.\sigma_{y}^{2}=100\right)$ the solution is not capillary dominated, as was discussed in figure $2\left(N_{b}=780\right)$ with saturation values around $\left\langle\widetilde{S}_{w}^{\text {init }}\right\rangle$, but rather presents a varying saturation profile (light blue curve).

\section{Results with entry pressure}

We now turn to study gravity segregation with $\mathrm{BC}$ capillary pressure, i.e. $P_{c}$ is given by (2.4) and (2.6). The main difference from the VG case is the capillary 


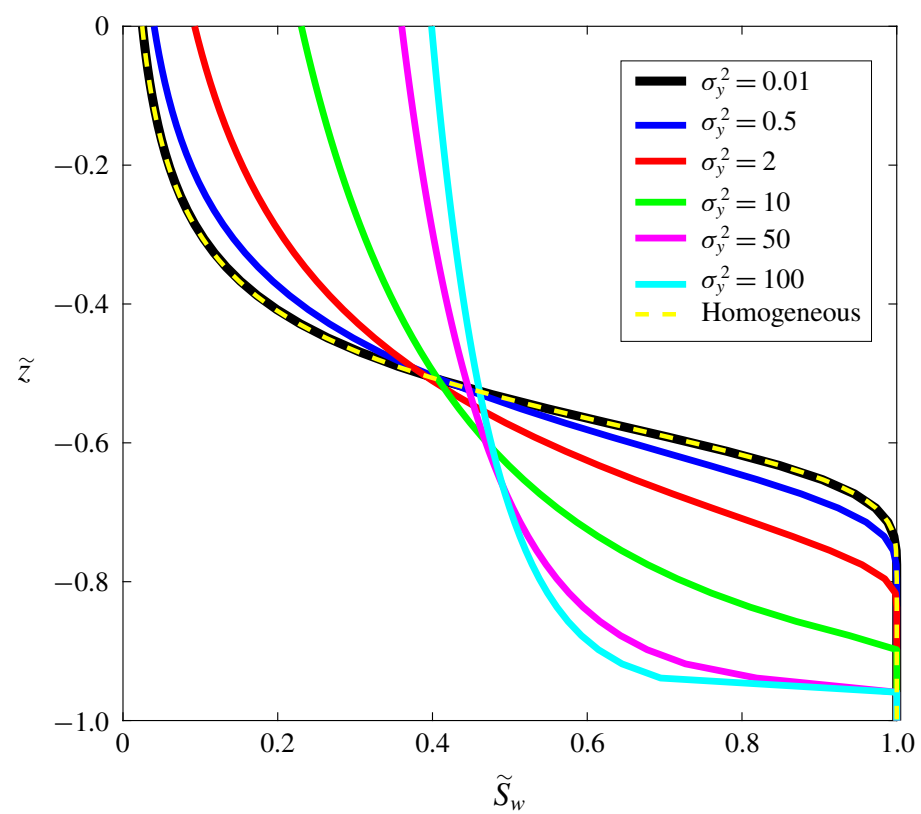

FIGURE 5. Ensemble mean saturation profiles for varying $\sigma_{y}^{2}$.

entry pressure, which is non-zero and changes spatially with permeability. Observing equation (3.3), we see that $\widetilde{S}_{w}=1$ is now obtained when $\widetilde{z}<\widetilde{P}_{e}-\widetilde{P}_{c}^{0}$, which is spatially varying with $\widetilde{k}$. This means that regions which are completely water saturated can exist at the same level $\widetilde{z}$ as regions with $\mathrm{CO}_{2}$. This does not occur in the VG case. The second and perhaps more significant impact of $\widetilde{P}_{e}$ is capillary entry trapping, which is not captured at all by the equilibrium analytical solution. During the dynamic migration of the fluids, regions with $\mathrm{CO}_{2}$ will not invade a fully water saturated region unless their capillary pressure exceeds the entry pressure of the fully saturated zone. This leads to an equilibrium solution in which some regions with large entry pressure remain with $\widetilde{S}_{w}=1$ since they are surrounded by zones of low capillary pressure (trapped water) or regions of small capillary pressure surrounded by $\widetilde{S}_{w}=1$ zones with large entry pressure (trapped $\mathrm{CO}_{2}$ ). Our numerical solution, however, includes the time evolution and incorporates capillary entry trapping (see Li (2011) for details).

Figure 6 presents results for $\mathrm{CO}_{2}$ saturation distribution in a slice through the domain centre for both analytical (panels $a, c, e$ ) and numerical (panels $b, d, f$ ) calculations. Parameters of $\lambda=2$ and uniform $\widetilde{S}_{\mathrm{CO}_{2}}^{\text {init }}=0.5$ are taken. Three values of $N_{b}$ are considered spanning from gravity to capillary dominated regimes, as was presented in figure 1 for the VG case. In fact, there is a similarity between the two figures since the parameters and the permeability field are the same, however, differences are apparent (particularly for panels $c$ and $d$ ) due to the different $\widetilde{P}_{c}$ functions. A comparison between analytical and numerical results in figure 6 shows much agreement, but also substantial differences. For example, in figures $6(b)$ and $6(d)$, there is trapped $\mathrm{CO}_{2}$ in the lower regions, which are completely water saturated according to the analytical solution. Errors are presented quantitatively in table 2, where for the capillary dominated case $\left(N_{b}=780\right.$, figure $\left.6(e, f)\right)$ they are very small. 
(a)

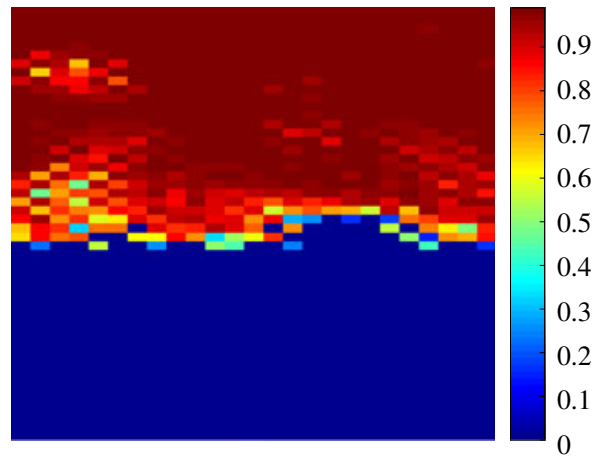

(c)

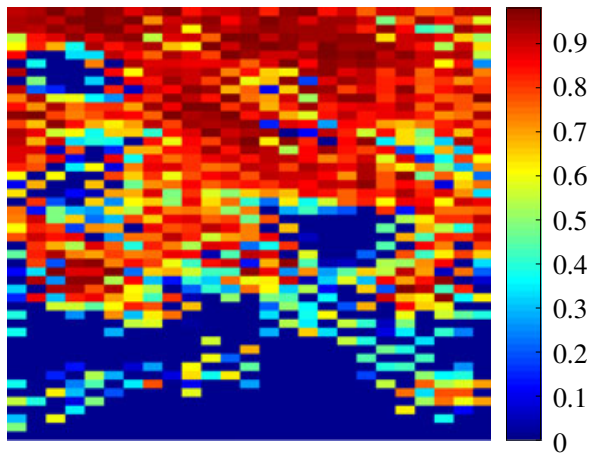

(e)

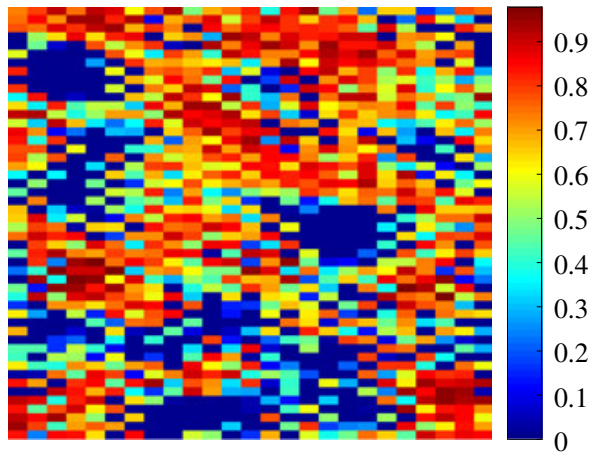

(b)

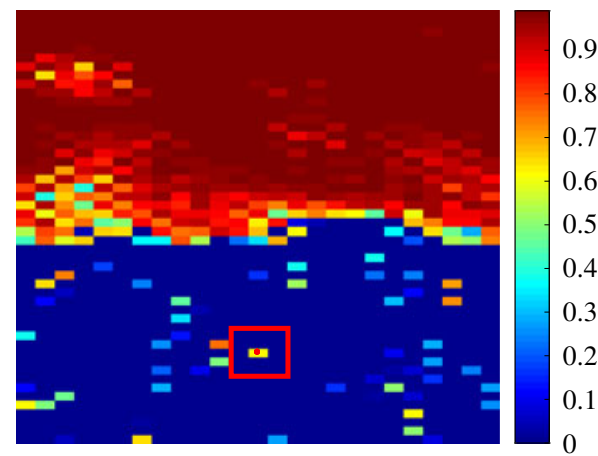

(d)

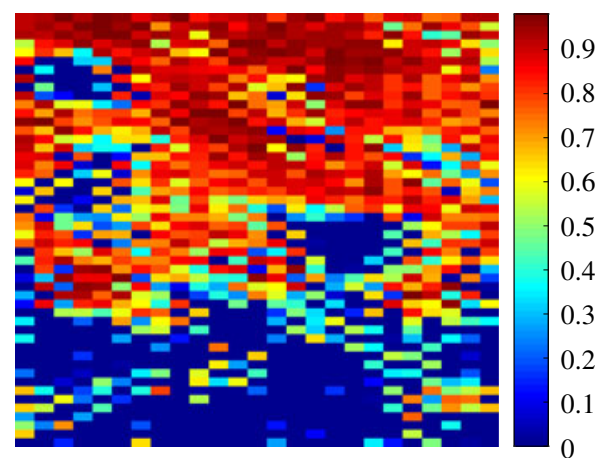

(f)

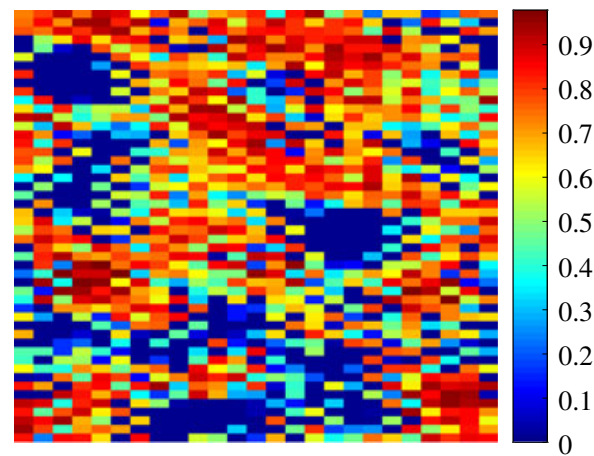

FIgURE 6. The $\mathrm{CO}_{2}$ saturation distribution in a vertical slice through the domain centre for $\mathrm{BC}$ capillary pressure. Plots $(a, c, e)$ are results for analytical solution while $(b, d, f)$ are numerical simulations. The first row of plots $(a, b)$ are results for $N_{b}=7.8$, the second row $(c, d)$ is for $N_{b}=78$ and the last row $(e, f)$ is for $N_{b}=780$.

Error increases with lower values of $N_{b}$ as the fully water saturated region becomes larger. This region is where the numerical solution presents entry pressure trapping of $\mathrm{CO}_{2}$ which cannot be predicted by the analytical solution.

To understand the entry pressure trapping presented in the numerical solution we plot the capillary pressure $\widetilde{P}_{c}$ corresponding to figure $6(b)$. This is presented in figure 7 . It is apparent that the top region, down to approximately $\widetilde{z}=-0.5$, has hydrostatic $\widetilde{P}_{c}$ variation in accordance to (3.1). In this region the analytical solution is in fairly good agreement with the numerical. However, below this region $\widetilde{P}_{c}$ changes 


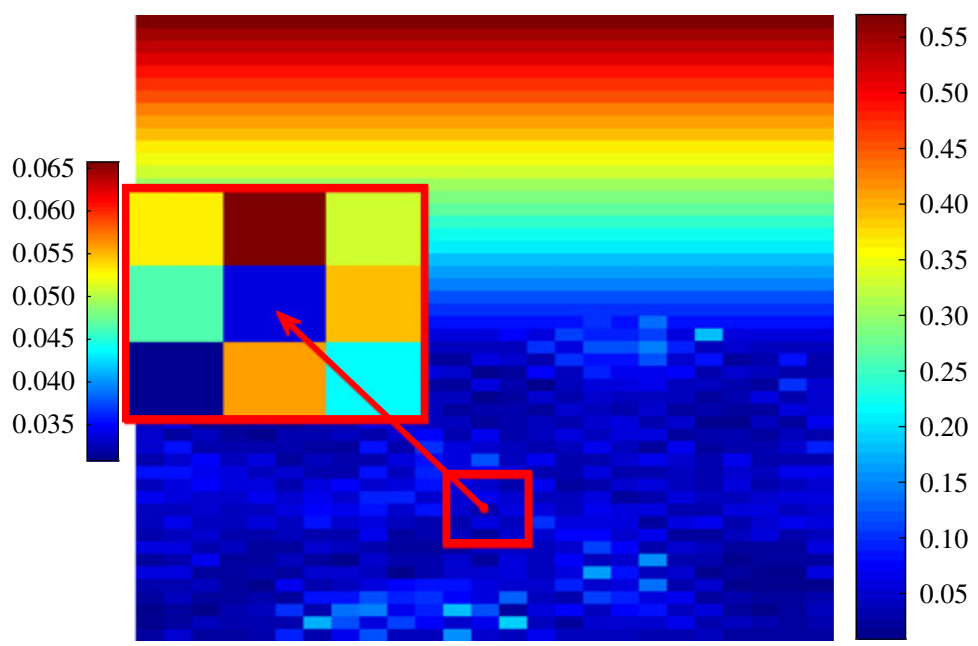

FIgURE 7. Dimensionless capillary pressure $\widetilde{P}_{c}$ in a vertical slice through the domain centre corresponding to figure $6(b)$. Inset: enlargement of a region with trapped $\mathrm{CO}_{2}$ as a result of entry pressure trapping (indicated in figure $6 b$ by a red rectangle).

\begin{tabular}{lccccccc}
\hline & \multicolumn{3}{c}{ Uniform initial } & & \multicolumn{3}{c}{ Bottom injection initial } \\
\cline { 2 - 3 } & $N_{b}=7.8$ & $N_{b}=78$ & $N_{b}=780$ & & $N_{b}=7.8$ & $N_{b}=78$ & $N_{b}=780$ \\
Mean error $(\langle E\rangle)$ & 0.022 & 0.016 & $7.9 \times 10^{-4}$ & & 0.043 & 0.089 & 0.037 \\
Error above $0.1\left(E_{0.1}\right)$ & $6.3 \%$ & $2.7 \%$ & $0.1 \%$ & & $11.5 \%$ & $27 \%$ & $8.6 \%$ \\
Error above $0.05\left(E_{0.05}\right)$ & $8.6 \%$ & $4.6 \%$ & $0.15 \%$ & & $14.1 \%$ & $31 \%$ & $19 \%$
\end{tabular}

TABLE 2. Error $E=\left|S_{w}^{\text {numerical }}(x, y, z)-S_{w}^{\text {analytical }}(x, y, z)\right|$ for different values of $N_{b}$ and considering two cases of initial conditions: uniform $\widetilde{S}_{\mathrm{CO}_{2}}^{\text {init }}=0.5$ and bottom injection of $\left\langle\widetilde{S}_{\mathrm{CO}_{2}}^{\text {init }}\right\rangle=0.1$ (figure $3 a$ ).

erratically from grid block to grid block. The majority of grid blocks in this lower region are fully water saturated and therefore capillary pressure is not well defined. The numerical simulator assigns the entry pressure to these grid blocks as virtual capillary pressure. Thus the observed erratic variation in the lower part of figure 7 is in fact the impact of permeability variation on entry pressure.

A grid block with trapped $\mathrm{CO}_{2}$ will have lower capillary pressure than all of its neighbouring grid blocks which results in a $\mathrm{CO}_{2}$ pressure gradient pointing towards this block $\left(p_{\mathrm{CO}_{2}}=p_{w}+P_{c}\right)$. Since the surrounding blocks are all fully water saturated, no $\mathrm{CO}_{2}$ can in fact migrate and the $\mathrm{CO}_{2}$ is trapped. An example for such a grid block is presented in the inset of figure 7, where the centre block consists of $\widetilde{S}_{w}=0.38$ and is surrounded by $\widetilde{S}_{w}=1$ neighbouring blocks. This enlarged region can also be observed in figure $6(b)$, marked with a red rectangle, showing the trapped $\mathrm{CO}_{2}$. Whether a grid block will contain trapped $\mathrm{CO}_{2}$ or not depends on the time evolution of capillary pressure and saturation, which makes it very difficult to estimate analytically using a steady-state approach. In the next section we will discuss a method for estimating this trapping. 


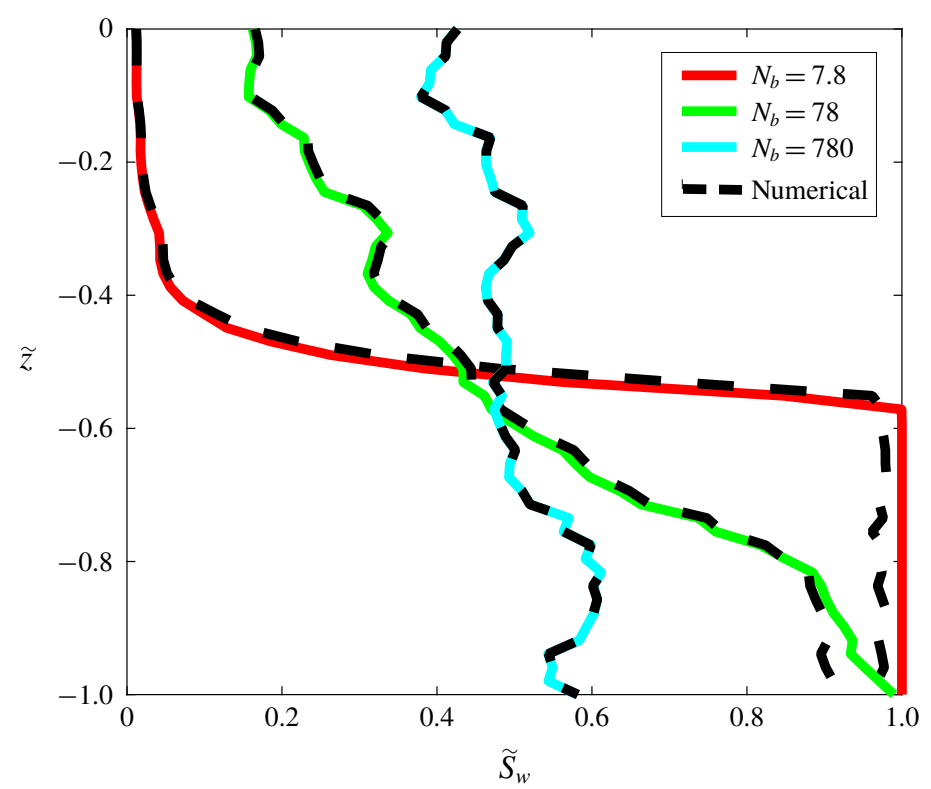

FIGURE 8. Horizontally averaged saturation as a function of height for different values of parameter $N_{b}$ considering BC capillary pressure and uniform $\widetilde{S}_{\mathrm{CO}_{2}}^{\text {init }}=0.5$. Analytical results (solid lines) given by (3.3) and (2.9) are compared with numerical simulation results (dashed lines).

Figure 8 presents results for the horizontally averaged water saturation profiles. The same conclusion that was discussed for figure 6 can be drawn here, i.e. a match between analytical and numerical results is seen for the case of $N_{b}=780$ while error is seen at the lower parts in cases $N_{b}=7.8,78$ for the analytical solution. Overall, the errors in the case of uniform $\widetilde{S}_{\mathrm{CO}_{2}}^{\text {init }}=0.5$ can be considered reasonable (see left-hand side of table 2) and the analytical solution is useful. This is not so for the next case, when we consider smaller amounts of initial $\mathrm{CO}_{2}$ saturation.

Figure 9 presents results for $\mathrm{CO}_{2}$ saturation distribution for the case of $\widetilde{S}_{\mathrm{CO}_{2}}^{\text {init }}$ as presented in figure $3(a)$, i.e. following injection of $\left\langle\widetilde{S}_{\mathrm{CO}_{2}}^{\text {init }}\right\rangle=0.1$ at the domain bottom. When entry pressure is included the equilibrium solution is no longer independent of the initial distribution $\widetilde{S}_{\mathrm{CO}_{2}}^{\text {init }}(x, y, z)$. Results of numerical simulations are strikingly different than the analytical solution here, particularly for the $N_{b}=78$ case (figure $9 c, d$ ). For all three values of $N_{b}$ the numerical results show significantly more $\mathrm{CO}_{2}$ at the lower parts of the domain. The right-hand side of table 2 presents errors for this case, showing the extent of the mismatch. The largest error is for the case with significant effect of both gravity and capillarity $\left(N_{b}=78\right)$, where $31 \%$ of the grid blocks have more than 0.05 error and $27 \%$ have error larger than 0.1 .

The capillary dominated case presented in figure $9(e, f)$ is of particular significance. One of the most popular methods for dealing with the computationally demanding $\mathrm{CO}_{2}$ migration models is to apply capillary limit upscaling (Mouche et al. 2010; Behzadi \& Alvarado 2012; Rabinovich et al. 2015; Rabinovich, Li \& Durlofsky 2016; Rabinovich 2018). This method entails assuming capillary equilibrium, uniform capillary pressure and subsequently the spatial distribution of $\widetilde{S}_{w}$ is obtained. Figure $9(e)$ is the saturation distribution under this assumption $\left(\widetilde{P}_{c}\right.$ is approximately 
(a)

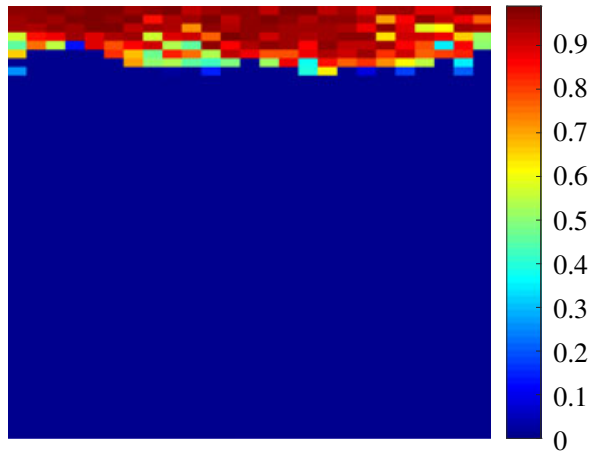

(c)

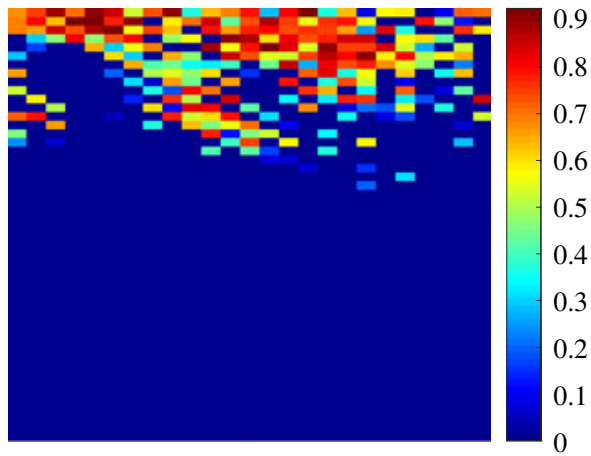

(e)

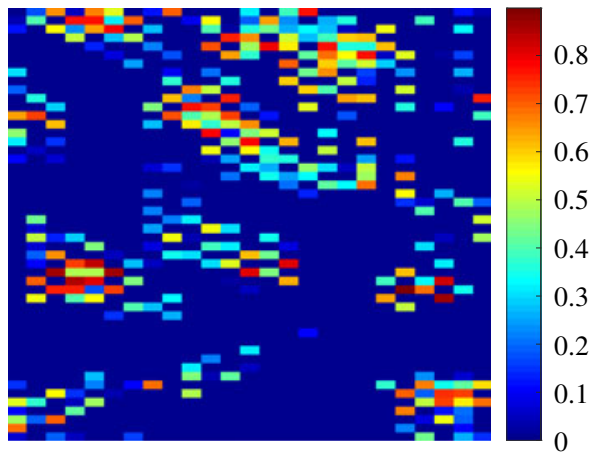

(b)

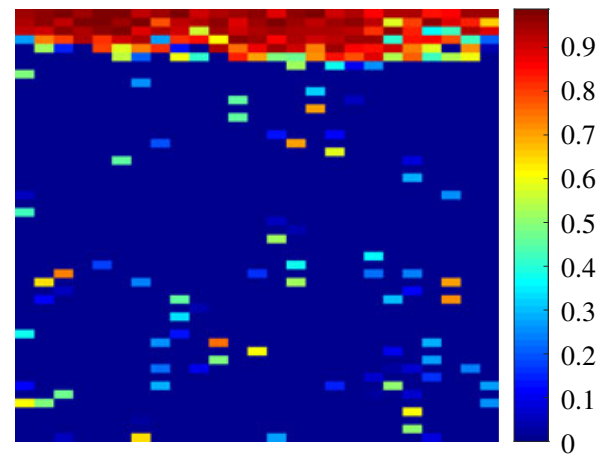

(d)

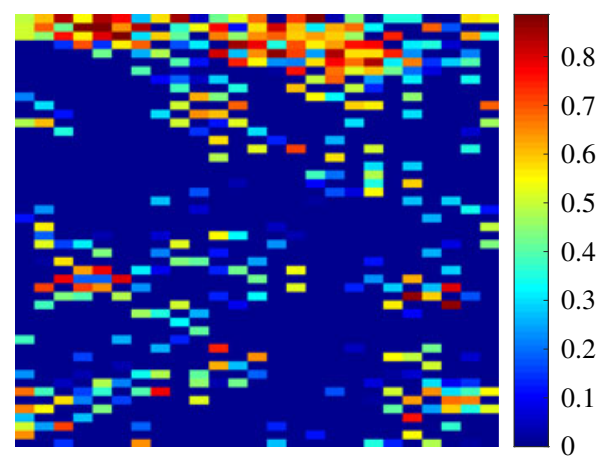

$(f)$

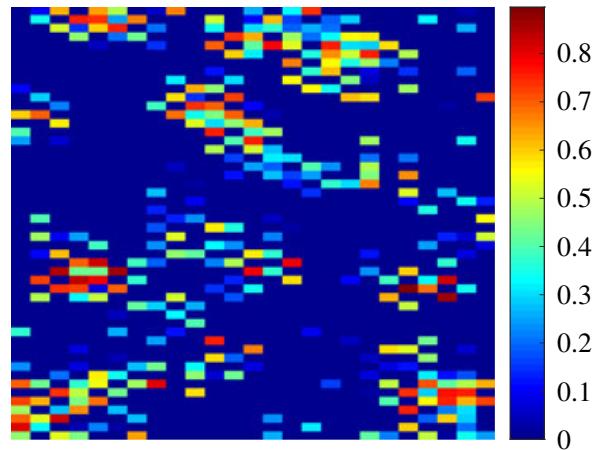

FIGURE 9. The $\mathrm{CO}_{2}$ saturation distribution in a vertical slice through the centre of the rectangular domain for $\mathrm{BC}$ capillary pressure and initial bottom injection of $\left\langle\widetilde{S}_{\mathrm{CO}_{2}}^{\text {init }}\right\rangle=0.1$. Plots $(a, c, e)$ are results for analytical solution while $(b, d, f)$ are numerical simulations. The first row of plots $(a, b)$ are results for $N_{b}=7.8$, the second row $(c, d)$ is for $N_{b}=78$ and the last row $(e, f)$ is for $N_{b}=780$.

constant in the domain). However, this assumption does not incorporate entry pressure trapping during migration, which leads to regions with non-uniform capillary pressure and additional trapped $\mathrm{CO}_{2}$ in lower regions, as observed in figure $9(f)$. This example leads us to the conclusion that under certain conditions, saturation derived from capillary limit assumptions may significantly deviate from the actual saturation distribution (see errors in table 2) and upscaling errors may follow. 


\subsection{Estimation of capillary entry pressure trapping}

We have previously established that the analytical solution in this work does not include the capillary entry pressure trapping and therefore may incur significant error. We now proceed by presenting a heuristic method for extending the solution of (3.3) and (2.9) to apply to cases with trapping. First, the main assumptions considered here will be detailed. In the previous results of $\S 5$ we show that the analytical solution for the case with $\left\langle\widetilde{S}_{\mathrm{CO}_{2}}^{\text {init }}\right\rangle=0.5$ is reasonably accurate while for $\left\langle\widetilde{S}_{\mathrm{CO}_{2}}^{\text {init }}\right\rangle=0.1$ there is significant error. We therefore aim to improve cases with small values of $\left\langle\widetilde{S}_{\mathrm{CO}_{2}}^{\text {init }}\right\rangle$, generally between 0 and 0.15 . This is also in line with applications of $\mathrm{CO}_{2}$ storage, where it is expected that the volumes of injected $\mathrm{CO}_{2}$ will be much smaller than the aquifer volume. Furthermore, we will limit our discussion to low-medium capillary numbers, i.e. approximately $N_{b}<33$. This should apply to many cases, particularly reservoir scale problems, in which $h$ is tens of metres $\left(N_{b} \propto 1 / h^{2}\right)$. Finally, we aim at estimating only the average saturation in each layer of the domain, i.e. obtaining $\left\langle\widetilde{S}_{w}\right\rangle_{h}(\widetilde{z})$, where \langle\rangle$_{h}$ denotes averaging horizontally. The full solution $\widetilde{S}_{w}(x, y, z)$ with entry trapping will not be estimated here.

A couple of tests are conducted on the solutions with entry pressure trapping to determine sensitivity to some parameters. First, we verify that the solution scales with $\Delta \rho g h^{2}$ so we can continue to work with the dimensionless $N_{b}$ parameter. This is carried out by conducting simulations with different $\Delta \rho$ but maintaining the same $N_{b}$ and observing no change in the solution. Then, the impact of different injection locations is tested and results are presented in figure $10(a-d)$ for the following configurations, respectively: uniform $\widetilde{S}_{\mathrm{CO}_{2}}^{\text {init }}$, uniform injection at the bottom domain boundary, injection at the centre point of the bottom boundary and injection at a bottom corner point of the domain.

Each of the four cases leads to a significantly different $\widetilde{S}_{\mathrm{CO}_{2}}^{\text {int }}$ distribution, however, all have the same average of $\left\langle\widetilde{S}_{\mathrm{CO}_{2}}^{\text {init }}\right\rangle=0.053$ and same problem parameters. It can be seen that the equilibrium solution does change with different initial conditions as opposed to the VG case which only depends on $\left\langle\widetilde{S}_{\mathrm{CO}_{2}}^{\text {init }}\right\rangle$. However, the change is relatively small and saturation distribution is quite similar in all four cases. We quantify the amount of trapped $\mathrm{CO}_{2}$ in each case, indicated at the bottom of each plot. It can be seen that a significant amount of $\mathrm{CO}_{2}$ (almost half) remains trapped via the entry pressure trapping mechanism, while the rest is in the confined space at the top four layers of the model. The relatively small impact of $\widetilde{S}_{\mathrm{CO}_{2}}^{\text {init }}$ distribution is seen considering the small difference in the trapped $\left\langle\widetilde{S}_{w}\right\rangle$ between the four cases. From here on we will develop the solution for uniform $\widetilde{S}_{\mathrm{CO}_{2}}^{\text {int }}$ with the understanding that it should apply to many other $\widetilde{S}_{\mathrm{CO}_{2}}^{\text {init }}$ distributions. Nevertheless, we only tested different types of bottom injection and other configurations such as top injection may present much less trapping as the $\mathrm{CO}_{2}$ will not reach the bottom layers.

The new solution, which includes entry pressure trapping, is given by

$$
\widetilde{S}_{w}= \begin{cases}{\left[\frac{1}{N_{b}} \sqrt{\frac{\widetilde{k}(x, y, z)}{\phi(x, y, z)}} \widetilde{P}_{c}^{\text {new }}(\widetilde{z})\right]^{-\lambda},} & \widetilde{P}_{e}<\widetilde{P}_{c}^{\text {new }} \\ 1, & \widetilde{P}_{e} \geqslant \widetilde{P}_{c}^{\text {new }},\end{cases}
$$


(a)

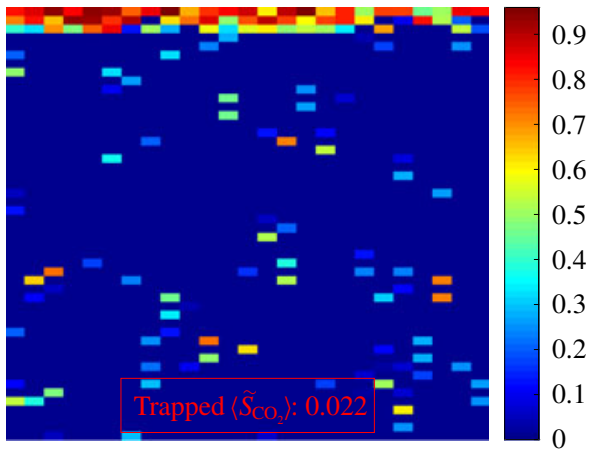

(c)

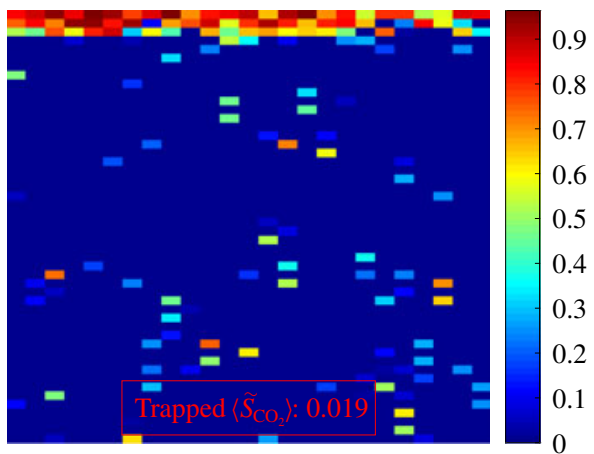

(b)

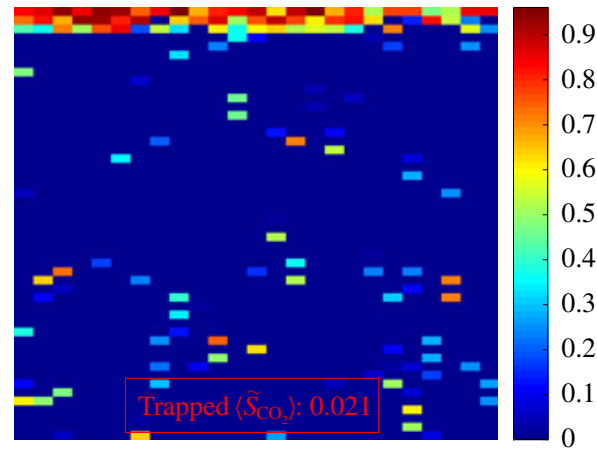

$(d)$



FIgURE 10. The $\mathrm{CO}_{2}$ saturation distribution in a vertical slice through the centre of the domain for different initial conditions: $(a)$ uniform, $(b)$ bottom uniform injection, (c) bottom centre point injection and $(d)$ bottom corner point injection. All cases consist of $\left\langle\widetilde{S}_{\mathrm{CO}_{2}}^{\text {init }}\right\rangle=0.053$.

where

$$
\widetilde{P}_{c}^{\text {new }}(\widetilde{z})= \begin{cases}\widetilde{P}_{c}^{0}+\widetilde{z}, & \widetilde{z} \geqslant \widetilde{z}^{\text {trap }} \\ \widetilde{P}_{c}^{\text {trap }}(\widetilde{z}), & \widetilde{z}<\widetilde{z}^{\text {trap }} \& ; \boldsymbol{x} \in \boldsymbol{x}_{\text {trap }} \\ 0, & \widetilde{z}<\widetilde{z}^{\text {trap }} \& \boldsymbol{x} \notin \boldsymbol{x}_{\text {trap }} .\end{cases}
$$

It represents the saturation distribution resulting from a new composite capillary pressure profile $\widetilde{P}_{c}^{\text {new }}$, which combines the original capillary pressure $\widetilde{P}_{c}^{0}+\widetilde{z}$ and the capillary pressure in the trapping region $\widetilde{P}_{c}^{\text {trap }}(\widetilde{z})$, to be defined in the following. Only certain regions, i.e. $\boldsymbol{x}_{\text {trap }}$ (to be defined in the following) which are prone to trapping are given $\widetilde{P}_{c}^{\text {trap }}(\widetilde{z})$ and all other regions have hydrostatic or zero $\widetilde{P}_{c}$. The transition between the two capillary pressure functions occurs at $\widetilde{Z}^{\text {trap }}$, which is simply the intersection of functions, i.e. $\widetilde{P}_{c}^{\text {trap }}\left(\widetilde{z}^{\text {trap }}\right)=\widetilde{P}_{c}^{0}+\widetilde{z}^{\text {trap }}$.

While the new modified solution is presented in (5.1), (5.2) as an analytical function, the calculation of $\widetilde{P}_{c}^{\text {trap }}(\widetilde{z})$ and $\boldsymbol{x}_{\text {trap }}$ will be described on a discretized domain. The first step is to determine the grid blocks which are prone to $\mathrm{CO}_{2}$ entry pressure trapping, i.e. those in $\boldsymbol{x}_{\text {trap }}$. They will be defined as grid blocks with entry pressure which is smaller than the entry pressure of all adjacent grid blocks, similar to what was observed in figure 7 . Figure $11(a)$ presents the entry pressure $\widetilde{P}_{e}$ pertaining to the case plotted in figure $9(b)$ with $N_{b}=7.8$. All of the grid blocks in $\boldsymbol{x}_{\text {trap }}$ are circled and shown to consist of small entry pressure. A comparison with figure $9(b)$ shows 
(a)

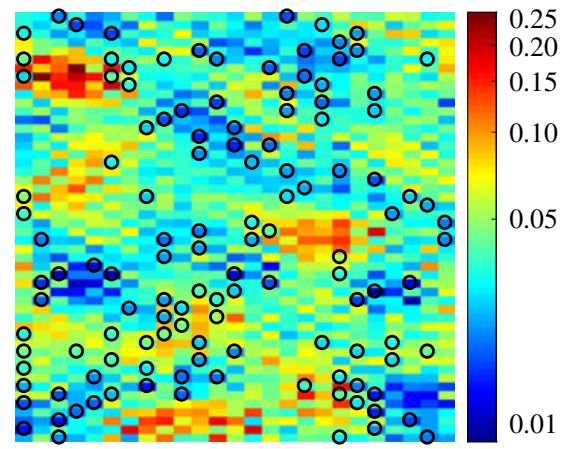

(b)

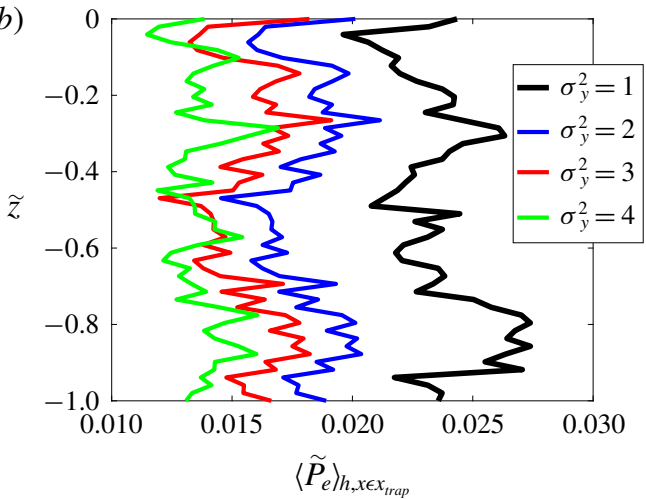

FIGURE 11. (a) Dimensionless capillary entry pressure $\widetilde{P}_{e}=\alpha C \sqrt{k / \phi} /(\Delta \rho g h)$ in a vertical slice through the centre of the domain corresponding to figure $9(b)$. Grid blocks in $\boldsymbol{x}_{\text {trap }}$, i.e. surrounded by lower $\widetilde{P}_{e}$, are circled. The colour bar is in a logarithmic scale. (b) Profiles of $\left\langle\widetilde{P}_{e}\right\rangle_{h}$, horizontally averaged only for $\boldsymbol{x} \in \boldsymbol{x}_{\text {trap }}$ considering different $k$ realizations with $\sigma_{y}^{2}$ as indicated in the legend.

that many of the circled grid blocks do have some trapped $\mathrm{CO}_{2}$ saturation, however, many others do not. Overall only around $50 \%$ of the grid blocks in $\boldsymbol{x}_{\text {trap }}$ have trapped $\mathrm{CO}_{2}$. Therefore, we cannot obtain high accuracy for predicting the blocks that will have entry pressure trapping. Nevertheless, the total number of blocks with trapping is matched very well with the total number of blocks in $\boldsymbol{x}_{\text {trap }}$. This allows for a good match in $\left\langle\widetilde{S}_{w}\right\rangle_{h}$ profiles as will be presented later on. We emphasize that the analysis was done on $N_{b}=7.8$ and for other $N_{b}$ values there will be a different number of grid blocks involved in trapping. This is not captured by $\boldsymbol{x}_{\text {trap }}$, which does not depend on $N_{b}$.

The capillary pressure $\widetilde{P}_{c}^{\text {trap }}(\widetilde{z})$ is defined using horizontally averaged entry pressure over all grid blocks in $\boldsymbol{x}_{\text {trap }}$, i.e. $\left\langle\widetilde{P}_{e}\right\rangle_{h, \boldsymbol{x} \in \boldsymbol{x}_{\text {trap }}}$. It is illustrated in figure $11(b)$ for four different $k$ realizations with changing variance $\sigma_{y}^{2}$. It can be seen that $\left\langle\widetilde{\boldsymbol{P}}_{e}\right\rangle_{h, \boldsymbol{x} \in \boldsymbol{x}_{\text {trap }}}$ decreases with increasing $\sigma_{y}^{2}$, as the entry pressure in blocks belonging to $\boldsymbol{x}_{\text {trap }}$ decreases due to the larger permeability extremes occurring when variance is larger. The impact of changing $N_{b}$ is simply a shift of the curves by a factor, since $\widetilde{P}_{e}$ is proportional to $N_{b}$. Altogether, the entry pressure trapping given by $\widetilde{P}_{c}^{\text {new }}$ in $(5.1)$ is rather heuristic and designed to give rough estimations. We therefore allow for an additional dimensionless factor $\widetilde{f}$ to correct for errors and $\widetilde{P}_{c}^{\text {trap }}$ is defined as follows

$$
\widetilde{P}_{c}^{\text {trap }}=\widetilde{f}\left(N_{b}, \sigma_{y}^{2}\right) \cdot\left\langle\widetilde{P}_{e}\right\rangle_{h, \boldsymbol{x} \in \boldsymbol{x}_{\text {trap }}} .
$$

The factor $\widetilde{f}$ is obtained by matching $\widetilde{S}_{w}$ from (5.1) with numerical solutions. For each simulation, we find $\widetilde{f}$ that minimized the objective function $\int_{\tilde{z}=-1}^{\widetilde{z}^{\text {trap }}} \mid\left\langle\widetilde{S}_{w}\right\rangle_{h}-$ $\left\langle\widetilde{S}_{w}\right\rangle_{h}^{\text {simulation }} \mid \mathrm{d} z$ and the results are plotted in figure 12 (filled circles). Altogether 30 numerical simulations were conducted for a range of $N_{b}$ and $\sigma_{y}^{2}$ values and the required $\widetilde{f}$ was found to behave logarithmically with $N_{b}$. A function was fitted to these 30 data points allowing to express the correction factor analytically as follows: 


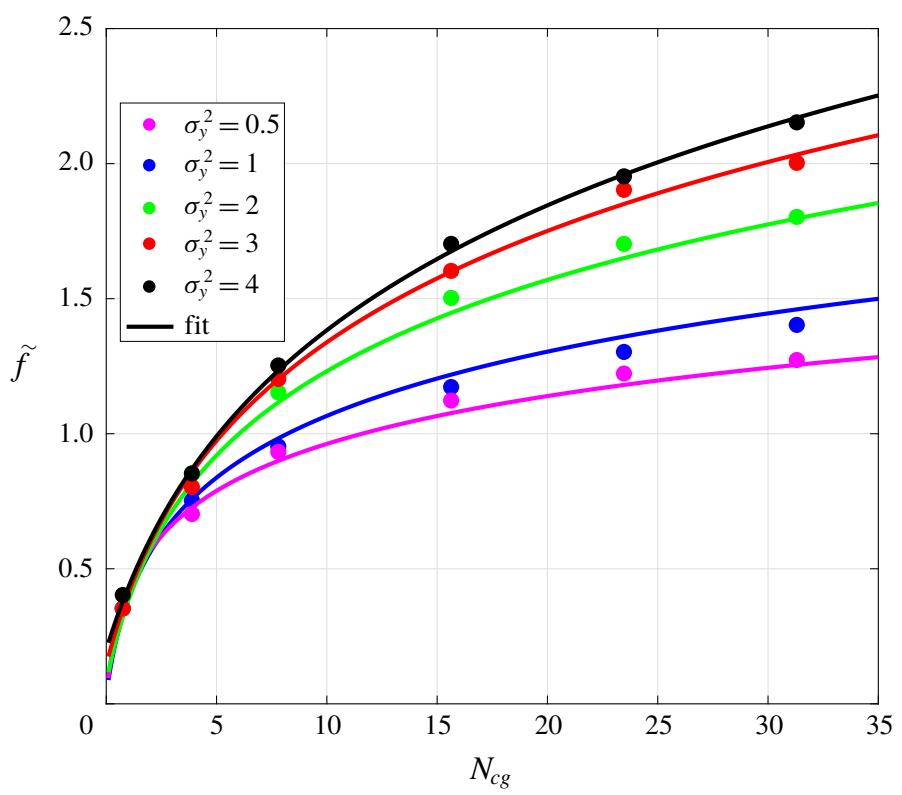

FIgURE 12. Correction factor $\widetilde{f}$ as a function of $N_{b}$ for different $k$ realizations with varying $\sigma_{y}^{2}$. A global fit given by (5.4) is plotted with colours corresponding to $\sigma_{y}^{2}$ values.

$$
\left.\begin{array}{c}
\tilde{f}=\beta \ln \left(0.65 N_{b}+\gamma\right)+\delta, \\
\beta=-0.0123 \sigma_{y}^{4}+0.213 \sigma_{y}^{2}+0.157, \\
\gamma=0.0328 \sigma_{y}^{4}+0.367 \sigma_{y}^{2}-0.017 \\
\delta=-0.0168 \sigma_{y}^{4}-0.159 \sigma_{y}^{2}+0.551 .
\end{array}\right\}
$$

Equation (5.4) is plotted in figure 12 (curves), where it can be seen that the function fits the data points fairly well. In the following we will test the solution given by (5.1)-(5.4) on a number of cases, some of which are not of the 30 cases used to construct the correction factor presented in figure 12. This will serve to demonstrate applicability.

Figure 13 presents results for numerical simulations (colour curves) alongside the new analytical solution incorporating trapping given by (5.1)-(5.4) (black curves). The plane averaged $\mathrm{CO}_{2}$ saturation is shown as a function of $\widetilde{z}$. Parameters for these cases are generally $N_{b}=7.8, \widetilde{S}_{\mathrm{CO}_{2}}^{\text {init }}=0.11$ (uniform), $\sigma_{y}^{2}=1$ and $\lambda=2$, except when other values are specified in the figure legends. In all cases the saturation profiles show a smooth top region in which the hydrostatic $\widetilde{P}_{c}$ is governing the solution and a lower region with erratic changes in which saturation is due to capillary entry trapping. The intersection of the two regions at $\widetilde{z}^{\text {trap }}$ can be seen. In all cases the analytical solution is observed to be roughly coinciding with the numerical results for the top region and adequately approximating the lower trapping region. Estimation is not necessarily accurate for each layer separately, however, both solutions fluctuate around the same average.

A quantitative comparison is presented in table 3 for each of the cases in figure 13. The results are the percentage of trapped $\mathrm{CO}_{2}$, i.e. ratios between trapped $\mathrm{CO}_{2}$ 
(a)

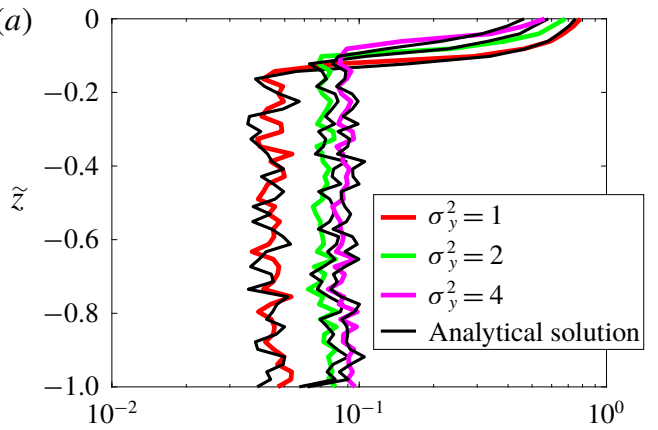

(c)

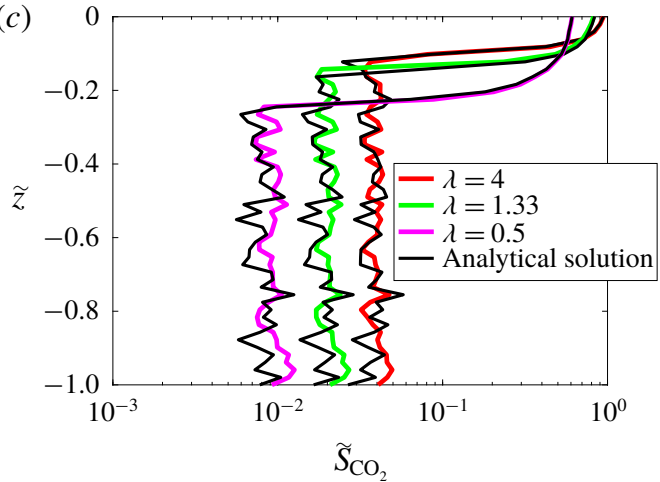

(b)

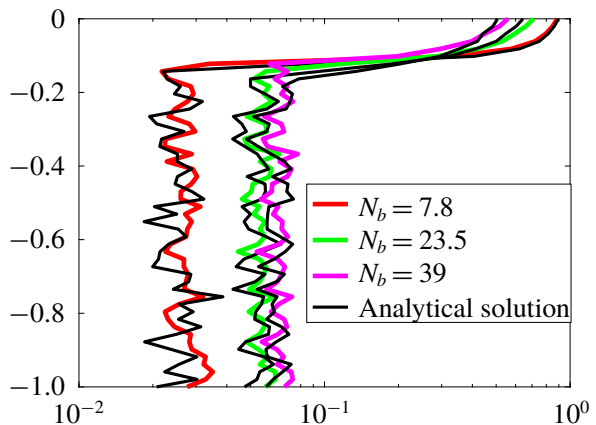

(d)

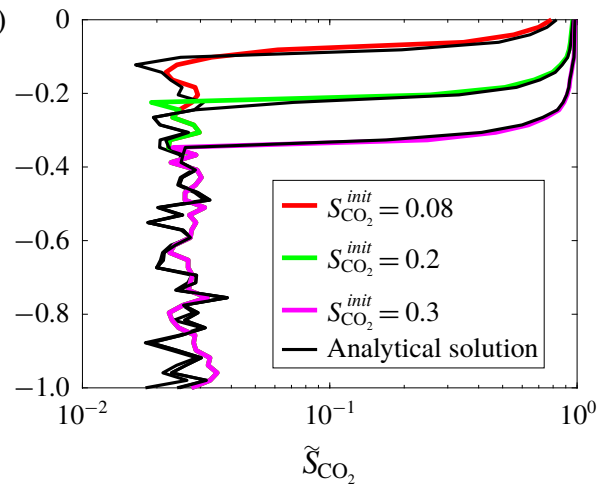

FIgURE 13. Horizontally averaged $\mathrm{CO}_{2}$ saturation as a function of $\widetilde{z}$ for varying (a) variance of $\log$ permeability, $(b)$ Bond number, $(c)$ initial saturation and $(d)$ capillary pressure parameter $(\lambda)$ in (2.6). Simulation results (coloured curves) are compared with analytical solution (black curves) and the $x$ axis is in a logarithmic scale.

Numerical Analytical

$\begin{array}{cccc} & \sigma_{y}^{2}=1 & 34.1 \% & 35.3 \% \\ \text { Figure 13(a) } & \sigma_{y}^{2}=2 & 59 \% & 56.7 \% \\ & \sigma_{y}^{2}=4 & 70 \% & 71 \% \\ & N_{b}=7.8 & 21.2 \% & 21.8 \% \\ \text { Figure 13(b) } & N_{b}=23.5 & 41.8 \% & 42.9 \% \\ & N_{b}=39 & 51 \% & 51.2 \% \\ & \widetilde{S}_{\mathrm{CO}_{2}}^{\text {init }}=0.08 & 26.8 \% & 29.9 \% \\ \text { Figure 13(c) } & \widetilde{S}_{\mathrm{CO}_{2}}^{\text {init }}=0.2 & 9.7 \% & 10.4 \% \\ & \widetilde{S}_{\mathrm{CO}_{2}}^{\text {init }}=0.3 & 5.7 \% & 6.1 \% \\ & \lambda=4 & 31.4 \% & 33 \% \\ \text { Figure 13(d) } & \lambda=1.33 & 14.3 \% & 15.8 \% \\ & \lambda=0.5 & 5.4 \% & 6.3 \%\end{array}$

TABLE 3. Comparison between numerical and analytical estimation of trapped $\mathrm{CO}_{2}$ for the cases presented in figure $13(a-d)$. Values in table represent the ratio between trapped $\mathrm{CO}_{2}$ and total $\mathrm{CO}_{2}$, i.e. $\left\langle\widetilde{S}_{\mathrm{CO}_{2}}\right\rangle \widetilde{z}<z^{\text {rap }} /\left\langle\widetilde{S}_{\mathrm{CO}_{2}}\right\rangle$. 
(defined as the $\mathrm{CO}_{2}$ in the region $\widetilde{z}<\widetilde{z}^{\text {trap }}$ ) and total $\mathrm{CO}_{2}$. In general, good agreement between the analytical and numerical results is seen.

Figure 13 allows us to investigate the dependence of the solution on various parameters. Figure 13(a), shows the increase in trapping when the variance of the $\log$ permeability field increases. This is due to an increase in entry pressure difference between blocks in $\boldsymbol{x}_{\text {trap }}$ and adjacent blocks, allowing for more capillary pressure build-up in $\boldsymbol{x}_{\text {trap }}$ blocks, i.e. more trapped $\mathrm{CO}_{2}$ saturation. In the analytical solution, this is a result of the factor $\widetilde{f}$ in (5.3), which increases with $\sigma_{y}^{2}$ (see figure 12). Figure 13(b) shows that trapping increases with larger $N_{b}$ and this is expected since increasing $N_{b}$ leads to more capillary effects, as seen in (2.6). Figure 13(d) shows the impact of initial saturation on $\left\langle\widetilde{S}_{w}\right\rangle_{h}$ profiles. While the top hydrostatic region is significantly affected by $\widetilde{S}_{\mathrm{CO}_{2}}^{\text {init }}$, the trapping zone does not change. This is because, in general, $\widetilde{S}_{\mathrm{CO}_{2}}^{\text {init }}$ does not play a role in the balance between capillary and gravity forces. However, if there is a region in which $\widetilde{S}_{\mathrm{CO}_{2}}^{\text {init }}$ is sufficiently small, it may reduce the trapping as there will be a lack of $\mathrm{CO}_{2}$ to remain trapped. In our case of mild entry pressure trapping $\left(N_{b}=0.1, \sigma_{y}^{2}=1\right)$ and uniformly distributed $\widetilde{S}_{\mathrm{CO}_{2}}^{\text {init }}$ there is sufficient initial saturation in all three cases presented. Only below $\widetilde{S}_{\mathrm{CO}_{2}}^{\text {init }}=0.05$ do we begin to observe a reduction in trapping.

Figure 13(c) presents the results for varying $\lambda$, which are rather interesting. Here, it can be seen that decreasing $\lambda$ decreases the amount of trapped $\mathrm{CO}_{2}$, despite the fact that capillary pressure will increase as seen in (2.6). In fact, the upper part of the curve shows that capillary pressure is increasing with smaller $\lambda$ as the smooth curves dip lower indicating more $\mathrm{CO}_{2}$ present in the lower parts of the domain. So we find that smaller $\lambda$ is generally associated with increased trapping in the hydrostatic (upper) part but a decrease in entry pressure trapping in the lower part. The reason is that while changing $\lambda$ will affect the $P_{c}$ curve, it does not impact the entry pressure. The reduction of entry pressure trapping is simply a result of mass conservation as there is more $\mathrm{CO}_{2}$ trapped in the upper parts in the hydrostatic region of the solution.

\subsection{Ensemble mean results}

We now present calculations for ensemble mean saturation profiles in the case of BC capillary pressure, i.e. including entry pressure trapping. The solution given by (3.10) does not incorporate entry-pressure trapping and therefore a correction must be applied, as done previously in $\$ 5.1$. We identify that the saturation ensemble average of trapping regions for cases of small $\left\langle\widetilde{S}_{\mathrm{CO}_{2}}^{\text {init }}\right\rangle$ and small to medium $N_{b}$ (cases of interest here) is constant. Therefore, we propose to model the top region with hydrostatic $P_{c}$ using equation (3.10) and the bottom, trapping region, using constant saturation. The solution is formulated as follows:

$$
\left\langle\widetilde{S}_{w}\right\rangle_{E}= \begin{cases}F_{E}\left(\widetilde{z}, \widetilde{P}_{c}^{0}, \sigma_{y}^{2}, \lambda, \widetilde{k}_{G}\right), & \widetilde{z} \geqslant \widetilde{z}^{*} \\ \widetilde{S}^{*}, & \widetilde{z}<\widetilde{z}^{*},\end{cases}
$$

where $F_{E}$ is given by (3.10). Height $\widetilde{z}^{*}$ is the elevation of the intersection between $F_{E}$ and $\widetilde{S}^{*}$, obtained by solving $F_{E}=\widetilde{S}^{*}$ and the constant $\widetilde{P}_{c}^{0}$ is obtained, as usual, by employing (2.9).

The saturation $\widetilde{S}^{*}$ is calculated as follows. We solve the problem for a single realization, either from numerical simulation or analytically via equations (5.1) and 
(5.2). We then find a constant value of capillary pressure $\widetilde{P}_{c}^{*}=\left(\widetilde{P}_{c}^{0}+\widetilde{z}\right) / N_{b}$ that when substituted into (3.3) results in a saturation profile $\left\langle\widetilde{S}_{w}\right\rangle_{h}$ matching the single realization solution. More specifically, we focus on matching the trapping region. Then, $\widetilde{S}^{*}$ is calculated by averaging over all possible permeabilities, i.e.

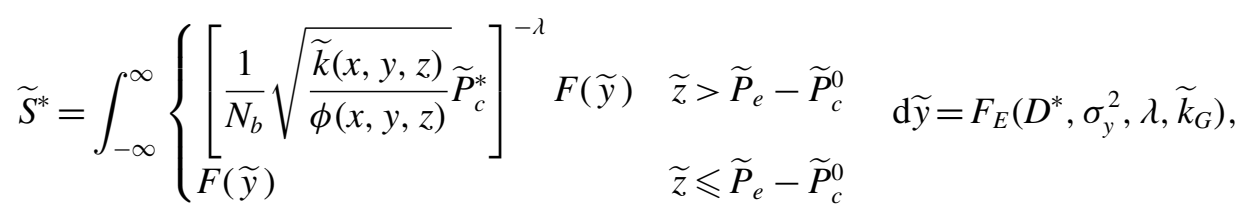

where $D^{*}=\widetilde{P}_{c}^{*} / \sqrt{\phi}$.

Results for equations (5.5) and (5.6) (denoted 'analytical mean') are plotted in figure $14(a, b)$ for four cases. Simulation results for five realizations are plotted for each case (thin curves) along with the average of all five simulations (dashed curves). There is significant scatter of the saturations with $\widetilde{z}$ in the trapping regions, particularly for the cases with anisotropic permeability structure. A comparison between results for anisotropic and isotropic permeability in each plot shows that the former have more variations with depth than the latter, also observed in figure 4 for VG capillary pressure. The overall trapping, however, is reduced in the anisotropic cases compared to the isotropic and this is most likely due to the fact that larger horizontal correlations promotes horizontal migration, which allows more paths to escape trapping.

Averaging saturation results from five realizations is clearly not enough to arrive at the accurate ensemble average, particularly for the highly varying anisotropic case. However, we have limited the number of simulations due to computational cost. Nevertheless, the results clearly show that the average of the realizations varies closely around the analytical ensemble mean for all four cases. This is particularly clear in the isotropic cases where the average of the realization saturations in the trapping region is almost constant with depth, coinciding with the analytical mean. Overall, the figures show the usefulness of the new ensemble average solution given by (5.5) and (5.6).

\section{Summary and conclusions}

This work derives an analytical solution to equilibrium gravity segregation with three-dimensional capillary heterogeneity. The fundamental solution assumes hydrostatic pressure variation and a $P_{c}\left(\phi, k, S_{w}\right)$ constitutive relation to arrive at a formula for $\widetilde{S}_{w}(x, y, z)$. Mass conservation is applied in order to obtain the constant $P_{c}^{0}$ appearing in the solution. The solution is exact and does not employ any approximations. It is therefore accurate for all cases without capillary entry pressure, i.e. for homogeneous media or capillary pressure curves in which $P_{c}\left(\widetilde{S}_{w}=1\right)=0$ (van Genuchten in our examples). The saturation distribution is analysed considering the impact of various dimensionless parameters: Bond number $N_{b}$, capillary pressure power $m$ or $\lambda$, permeability distribution $\widetilde{k}(x, y, z)$ and average initial saturation $\left\langle\widetilde{S}_{w}^{\text {init }}\right\rangle$. Validations of numerical simulators for problems with 3-D capillary heterogeneity have hardly been published (Hoteit \& Firoozabadi 2008) and the new solution could be very useful for such validation.

The case of $P_{c}$ with entry pressure $P_{c}\left(\widetilde{S}_{w}=1\right) \neq 0$ (Brooks-Corey in our examples) is investigated by comparing the analytical and numerical solutions. The entry pressure 

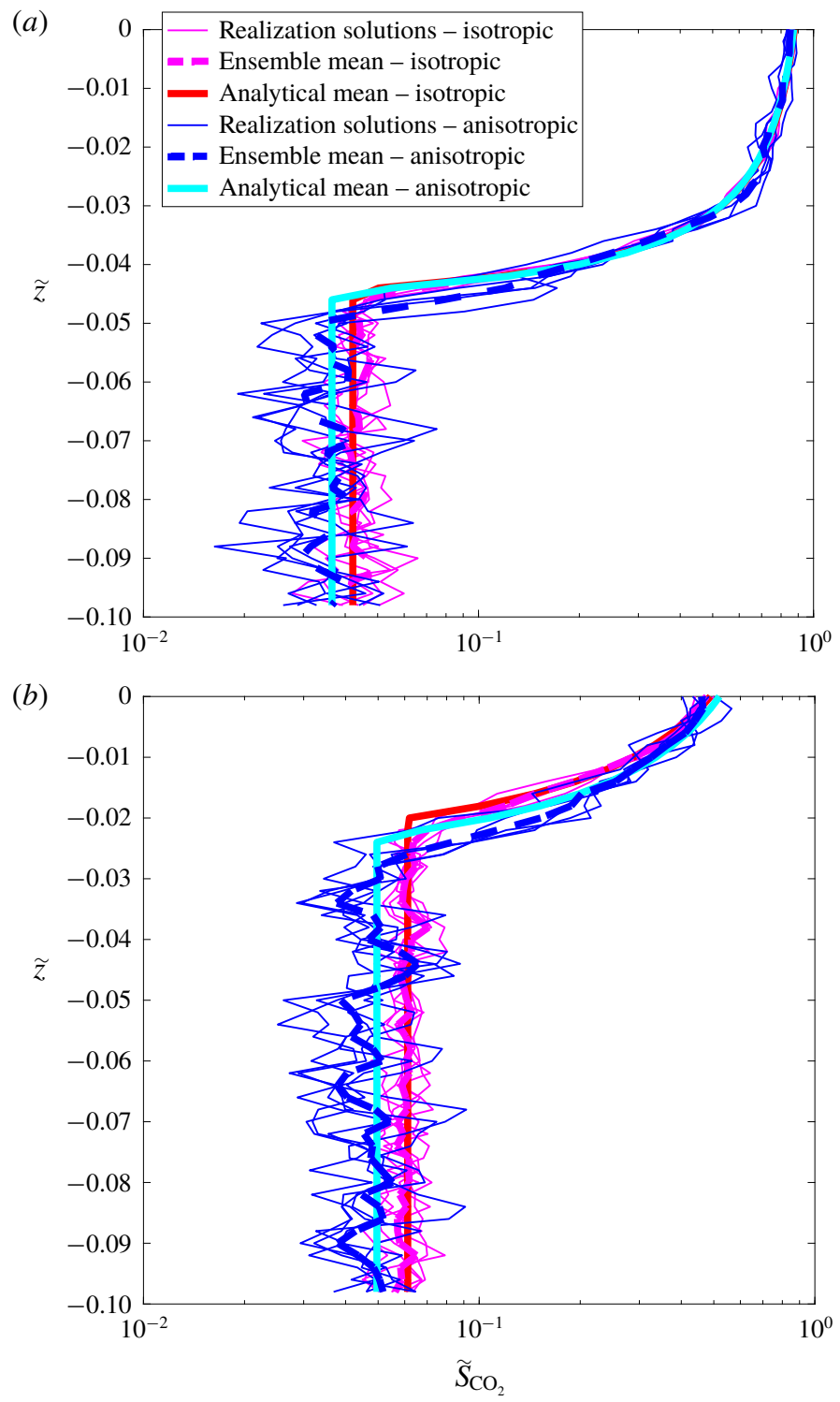

FIGURE 14. Horizontally averaged $\mathrm{CO}_{2}$ saturation $\left\langle\widetilde{S}_{\mathrm{CO}_{2}}\right\rangle_{h}$ as a function of $\widetilde{z}$ for five different realizations, ensemble mean of the five realizations and analytical solution (equations (5.5) and (5.6)) for $(a) N_{b}=23.5, S_{\mathrm{CO}_{2}}^{\text {init }}=0.3$ and $(b) N_{b}=39, S_{\mathrm{CO}_{2}}^{\text {init }}=0.11$.

trapping mechanism is explained with examples illustrated using numerical results. It is shown that for large $\widetilde{S}_{w}^{\text {init }}$ uniformly distributed in the domain, the analytical solution does not estimate the entry pressure trapping but errors are reasonable and it is still applicable. However, for small $\left\langle\widetilde{S}_{w}^{\text {init }}\right\rangle$, which is relevant to $\mathrm{CO}_{2}$ storage applications in which a small volume of non-wetting phase is injected in the lower part of an aquifer, the errors are very large. This has an important implication regarding approximations which neglect entry pressure trapping, often used in $\mathrm{CO}_{2}$ 
storage modelling. A modification to the analytical solution is presented to account for entry pressure trapping. It is based on a heuristic formula, obtained by matching the numerical results and applies to a limited range of Bond numbers, i.e. $N_{b}<33$. The modified solution is shown to produce accurate estimates of the overall trapped $\mathrm{CO}_{2}$ and it is used to investigate the impact of different parameters on trapping.

The solutions for cases with and without entry pressure trapping are extended to consider random log-normally distributed $k$. The expected value of the saturation is derived analytically for the case without entry pressure trapping to arrive at a formula depending on $N_{b} \sqrt{\phi}, m, \sigma_{y}^{2}, \ln k_{G}$ and $\left\langle\widetilde{S}_{w}^{\text {init }}\right\rangle$. We show that solutions for realizations of $k$ with the same $\sigma_{y}^{2}$ and $k_{G}$ are all varying within a small range around the ensemble mean solution $\left\langle\widetilde{S}_{w}\right\rangle_{E}$. This leads to the conclusion that for a large enough sample size of permeability measurements the average solution can be used with sufficient accuracy to predict gravity-capillary equilibrium of two fluids.

\section{Acknowledgements}

This work was supported by the Israeli Ministry of National Infrastructure, Energy and Water Resources.

\section{Declaration of interests}

The authors report no conflict of interest.

\section{Appendix A. Derivation of $\left\langle\widetilde{S}_{w}\right\rangle_{E}$ for the VG model}

Starting from (3.7), we now present the derivation of (3.8), focusing on the case of $\widetilde{z}>\widetilde{P}_{c}^{0}$ (since the solution is simply 1 for $\widetilde{z}<\widetilde{P}_{c}^{0}$ ). The expression in the integral of (3.7) can be expanded using a Taylor (binomial) series expansion as follows:

$$
\left(A \exp \left[\frac{\tilde{y}}{2(1-m)}\right]+1\right)^{-m}=\sum_{n=0}^{\infty}\left(\begin{array}{c}
-m \\
n
\end{array}\right) A^{n} \exp \left[\frac{n \widetilde{y}}{2(1-m)}\right]
$$

for $A \exp [\tilde{y} /(2(1-m))]<1$, and

$$
\left(A \exp \left[\frac{\tilde{y}}{2(1-m)}\right]+1\right)^{-m}=\sum_{n=0}^{\infty}\left(\begin{array}{c}
-m \\
n
\end{array}\right) A^{-(n+m)} \exp \left[\frac{(n+m) \widetilde{y}}{2(m-1)}\right],
$$

for $A \exp [\widetilde{y} /(2(1-m))]>1$, where $\left(\begin{array}{c}-m \\ n\end{array}\right)$ are the binomial coefficients. Substituting (A 1) and (A 2) into (3.7) and integrating separately on $\left(-\infty, \widetilde{y}_{L}\right)$ and $\left(\widetilde{y}_{L}, \infty\right)$ we arrive at

$$
\begin{aligned}
\left\langle\widetilde{S}_{w}\right\rangle_{E}= & \sum_{n=0}^{\infty}\left(\begin{array}{c}
-m \\
n
\end{array}\right)\left\{A^{n} \int_{-\infty}^{\widetilde{y_{L}}} \exp \left[\frac{n \widetilde{y}}{2(1-m)}\right] F(\widetilde{y}) \mathrm{d} \widetilde{y}\right. \\
& \left.+A^{-(n+m)} \int_{\widetilde{y}_{L}}^{\infty} \exp \left[\frac{(n+m) \widetilde{y}}{2(1-m)}\right] F(\widetilde{y}) \mathrm{d} \widetilde{y}\right\},
\end{aligned}
$$

where $\widetilde{y}_{L}=2(1-m) \ln A$. Substituting (3.5) into (A 3) and solving the integrals we arrive at the final expression presented in (3.8). 


\section{REFERENCES}

Andersen, O., Gasda, S. E. \& Nilsen, H. M. 2015 Vertically averaged equations with variable density for $\mathrm{CO}_{2}$ flow in porous media. Trans. Porous Med. 107 (1), 95-127.

Bedrikovetsky, P. 2013 Mathematical Theory of Oil and Gas Recovery: With Applications to ex-USSR Oil and Gas Fields, vol. 4, chap. 25. Springer.

Behzadi, H. \& Alvarado, V. 2012 Upscaling of upward $\mathrm{CO}_{2}$ migration in 2D system. Adv. Water Resour. 46, 46-54.

Brooks, R. H. \& Corey, A. T. 1966 Properties of porous media affecting fluid flow. J. Irrigation Drainage Division 92 (2), 61-90.

Bryant, S. L., Lakshminarasimhan, S. \& Pope, G. A. 2006 Buoyancy-dominated multiphase flow and its impact on geological sequestration of $\mathrm{CO}_{2}$. In SPE/DOE Symposium on Improved Oil Recovery. Society of Petroleum Engineers.

CAO, H. 2002, Development of techniques for general purpose simulators. PhD thesis, Stanford University, Stanford, CA, USA.

Carruthers, D. J. \& VAn WijngaArden, M. 2000 Modelling viscous-dominated fluid transport using modified invasion percolation techniques. J. Geochem. Explor. 69, 669-672.

Cheng, K. B., Rabinovich, A. \& Dagan, G. 2019 Stochastic modeling of oscillatory pumping in heterogeneous aquifers with application to Boise aquifer test. J. Hydrol. 569, 278-290.

Deng, H., Stauffer, P. H., Dai, Z., JiAO, Z. \& Surdam, R. C. 2012 Simulation of industrial-scale $\mathrm{CO}_{2}$ storage: Multi-scale heterogeneity and its impacts on storage capacity, injectivity and leakage. Intl J. Greenh. Gas Control 10, 397-418.

Dentz, M. \& TARTakovsky, D. M. 2009 Abrupt-interface solution for carbon dioxide injection into porous media. Trans. Porous Med. 79 (1), 15.

Deutsch, C. V. \& Journel, A. G. 1992 GSLIB: Geostatistical Software Library and User's Guide. Oxford University Press.

Donato, G. D., Tavassoli, Z. \& Blunt, M. J. 2006 Analytical and numerical analysis of oil recovery by gravity drainage. J. Petrol. Sci. Engng 54 (1), 55-69.

DongXiaO, Z. \& TCHElepI, H. 1999 Stochastic analysis of immiscible two-phase flow in heterogeneous media. SPE J. 4 (04), 380-388.

Dullien, F. A. L. 2012 Porous Media: Fluid Transport and Pore Structure. Academic.

Gasda, S. E., Nilsen, H. M. \& DAHLE, H. K. 2013 Impact of structural heterogeneity on upscaled models for large-scale $\mathrm{CO}_{2}$ migration and trapping in saline aquifers. Adv. Water Resour. 62, $520-532$.

Gasda, S. E., Nordbotten, J. M. \& Celia, M. A. 2009 Vertical equilibrium with sub-scale analytical methods for geological $\mathrm{CO}_{2}$ sequestration. Comput. Geosci. 13 (4), 469-481.

VAN GenuChten, M. T. 1980 A closed-form equation for predicting the hydraulic conductivity of unsaturated soils. Soil Sci. Soc. Am. J. 44 (5), 892-898.

Gershenzon, N. I., Soltanian, M., JR, R. W. R. \& Dominic, D. F. 2014 Influence of small scale heterogeneity on $\mathrm{CO}_{2}$ trapping processes in deep saline aquifers. Energy Procedia 59, $166-173$.

Golding, M. J. \& HupperT, H. E. 2010 The effect of confining impermeable boundaries on gravity currents in a porous medium. J. Fluid Mech. 649, 1-17.

Golding, M. J., Neufeld, J. A., Hesse, M. A. \& Huppert, H. E. 2011 Two-phase gravity currents in porous media. J. Fluid Mech. 678, 248-270.

Green, C. P. \& EnNis-King, J. 2013 Residual trapping beneath impermeable barriers during buoyant migration of $\mathrm{CO}_{2}$. Trans. Porous Med. 98 (3), 505-524.

Hagoort, J. 1980 Oil recovery by gravity drainage. Soc. Petrol. Engng J. 20 (03), 139-150.

Haldorsen, H. H., BRAnd, P. J. \& Macdonald, C. J. 1987 Review of the stochastic nature of reservoirs. In Conference Proceedings of Mathematics in Oil Production. European Association of Geoscientists \& Engineers.

Han, W. S., LeE, S.-Y., Lu, C.\& MCPherson, B. J. 2010 Effects of permeability on $\mathrm{CO}_{2}$ trapping mechanisms and buoyancy-driven $\mathrm{CO}_{2}$ migration in saline formations. Water Resour. Res. 46 (7), W07510.

Hesse, M. A., OrR, F. M. \& TChelepi, H. A. 2008 Gravity currents with residual trapping. J. Fluid Mech. 611, 35-60. 
Hesse, M. A., Tchelepi, H. A., Cantwel, B. J. \& OrR, F. M. 2007 Gravity currents in horizontal porous layers: transition from early to late self-similarity. J. Fluid Mech. 577, 363-383.

Hesse, M. A. \& Woods, A. W. 2010 Buoyant dispersal of $\mathrm{CO}_{2}$ during geological storage. Geophys. Res. Lett. 37 (1), L01403.

Hilfer, R. 2006 Capillary pressure, hysteresis and residual saturation in porous media. Physica A 359, 119-128.

Hoteit, H. \& Firoozabadi, A. 2008 Numerical modeling of two-phase flow in heterogeneous permeable media with different capillarity pressures. Adv. Water Resour. 31 (1), 56-73.

Hovorka, S. D., Doughty, C., Benson, S. M., Pruess, K. \& Knox, P. R. 2004 The impact of geological heterogeneity on $\mathrm{CO}_{2}$ storage in brine formations: a case study from the Texas gulf coast. Geol. Soc. Lond. Spec. Publ. 233 (1), 147-163.

IDE, S. T., Jessen, K. JR \& ORR, F. M. 2007 Storage of $\mathrm{CO}_{2}$ in saline aquifers: effects of gravity, viscous, and capillary forces on amount and timing of trapping. Intl J. Greenh. Gas Control 1 (4), 481-491.

IoAnnidis, M. A., Chatzis, I. \& Dullien, F. A. L. 1996 Macroscopic percolation model of immiscible displacement: Effects of buoyancy and spatial structure. Water Resour. Res. 32 (11), 3297-3310.

Juanes, R., MacMinn, C. W. \& Szulczewski, M. L. 2010 The footprint of the $\mathrm{CO}_{2}$ plume during carbon dioxide storage in saline aquifers: storage efficiency for capillary trapping at the basin scale. Trans. Porous Med. 82 (1), 19-30.

JuAnes, R., SPiteri, E. J., ORR, F. M. JR \& Blunt, M. J. 2006 Impact of relative permeability hysteresis on geological $\mathrm{CO}_{2}$ storage. Water Resour. Res. 42 (12), W12418.

Krause, M. H. 2012 Modeling and investigation of the influence of capillary heterogeneity on relative permeability. In SPE Annual Technical Conference and Exhibition, San Antonio, Texas, SPE-160909-STU. Society of Petroleum Engineers.

Krevor, S., Blunt, M. J., Benson, S. M., Pentland, C. H., Reynolds, C., Al-Menhali, A. \& NIU, B. 2015 Capillary trapping for geologic carbon dioxide storage-from pore scale physics to field scale implications. Intl J. Greenh. Gas Control 40, 221-237.

Krevor, S. C., Pini, R., LI, B.\& Benson, S. M. 2011 Capillary heterogeneity trapping of $\mathrm{CO}_{2}$ in a sandstone rock at reservoir conditions. Geophys. Res. Lett. 38 (15), L15401.

Krevor, S. C., Pini, R., Zuo, L. \& Benson, S. M. 2012 Relative permeability and trapping of $\mathrm{CO}_{2}$ and water in sandstone rocks at reservoir conditions. Water Resour. Res. 48 (2), W02532.

Kuo, C.-W. \& Benson, S. M. 2015 Numerical and analytical study of effects of small scale heterogeneity on $\mathrm{CO}_{2}$ /brine multiphase flow system in horizontal corefloods. Adv. Water Resour. 79, $1-17$.

LEE, S. T. 1989 Capillary-gravity equilibria for hydrocarbon fluids in porous media. In SPE Annual Technical Conference and Exhibition. Society of Petroleum Engineers.

Lenormand, R., ZARCone, C. \& SARR, A. 1983 Mechanisms of the displacement of one fluid by another in a network of capillary ducts. J. Fluid Mech. 135, 337-353.

LI, B. \& BENSON, S. M. 2015 Influence of small-scale heterogeneity on upward $\mathrm{CO}_{2}$ plume migration in storage aquifers. Adv. Water Resour. 83, 389-404.

LI, B. 2011 Including fine-scale capillary heterogeneity in modeling the flow of $\mathrm{CO}_{2}$ and brine in reservoir cores. PhD thesis, Stanford University.

Loubens, R. D. \& RAMAKRishnan, T. S. 2011 Analysis and computation of gravity-induced migration in porous media. J. Fluid Mech. 675, 60-86.

MacMinn, C. W. \& JUANES, R. 2009 Post-injection spreading and trapping of $\mathrm{CO}_{2}$ in saline aquifers: impact of the plume shape at the end of injection. Comput. Geosci. 13 (4), 483.

Malekzadeh, F. A., Heidari, R. \& Dusseault, M. 2017 An analytical solution for capillary gravity drainage with dominant viscous forces. Trans. Porous Med. 118 (3), 417-434.

Montel, F., Bickert, J., Lagisquet, A.\& Galliéro, G. 2007 Initial state of petroleum reservoirs: a comprehensive approach. J. Petrol. Sci. Engng 58 (3-4), 391-402.

Mouche, E., HAYeK, M. \& MüGler, C. 2010 Upscaling of $\mathrm{CO}_{2}$ vertical migration through a periodic layered porous medium: the capillary-free and capillary-dominant cases. Adv. Water Resour. 33 (9), 1164-1175. 
Nilsen, H. M., Lie, K.-A. \& Andersen, O. 2016 Fully-implicit simulation of vertical-equilibrium models with hysteresis and capillary fringe. Comput. Geosci. 20 (1), 49-67.

Nooruddin, H. A. \& Blunt, M. J. 2018 Large-scale invasion percolation with trapping for upscaling capillary-controlled Darcy-scale flow. Trans. Porous Med. 121 (2), 479-506.

Nordbotten, J. M. \& Celia, M. A. 2006 Similarity solutions for fluid injection into confined aquifers. J. Fluid Mech. 561, 307-327.

Nordbotten, J. M. \& Celia, M. A. 2011 Geological Storage of $\mathrm{CO}_{2}$ : Modeling Approaches for Large-scale Simulation. Wiley.

Nordbotten, J. M. \& DAhle, H. K. 2010 Impact of capillary forces on large-scale migration of $\mathrm{CO}_{2}$. In XVIII International Conference on Water Resources. CMWR.

Nordbotten, J. M. \& Dahle, H. K. 2011 Impact of the capillary fringe in vertically integrated models for $\mathrm{CO}_{2}$ storage. Water Resour. Res. 47 (2), W02537.

Oldenburg, C. M., Mukhopadhyay, S. \& Cihan, A. 2016 On the use of Darcy's law and invasion-percolation approaches for modeling large-scale geologic carbon sequestration. Greenhouse Gases: Sci. Technol. 6 (1), 19-33.

Pentland, C. H., El-Maghraby, R., Iglauer, S. \& Blunt, M. J. 2011 Measurements of the capillary trapping of super-critical carbon dioxide in Berea sandstone. Geophys. Res. Lett. 38 (6), L06401.

Perrin, J. C. \& Benson, S. M. 2010 An experimental study on the influence of sub-core scale heterogeneities on $\mathrm{CO}_{2}$ distribution in reservoir rocks. Trans. Porous Med. 82 (1), 93-109.

Pinder, G. F. \& Gray, W. G. 2008 Essentials of Multiphase Flow and Transport in Porous Media. Wiley.

Pini, R. \& BEnson, S. M. 2017 Capillary pressure heterogeneity and hysteresis for the supercritical $\mathrm{CO}_{2}$ /water system in a sandstone. Adv. Water Resour. 108, 277-292.

RABINOVICH, A. 2018 Analytical corrections to core relative permeability for low-flow-rate simulation. SPE J. 23 (05), 1-851.

Rabinovich, A., DAGAn, G. \& Miloh, T. 2012 Boundary effects on effective conductivity of random heterogeneous media with spherical inclusions. Phys. Rev. E 86 (4), 046601.

Rabinovich, A., ItThisawatpan, K. \& Durlofsky, L. J. 2015 Upscaling of $\mathrm{CO}_{2}$ injection into brine with capillary heterogeneity effects. J. Petrol. Sci. Engng 134, 60-75.

RABinovich, A., Li, B.\& DURlofsKy, L. J. 2016 Analytical approximations for effective relative permeability in the capillary limit. Water Resour. Res. 52 (10), 7645-7667.

Remy, N., Boucher, A. \& WU, J. 2009 Applied Geostatistics with SGeMS: A User's Guide. Cambridge University Press.

SAADATPOOR, E., Bryant, S. L. \& SEPEHRnoori, K. 2011 Effect of upscaling heterogeneous domain on $\mathrm{CO}_{2}$ trapping mechanisms. Energy Procedia 4, 5066-5073.

Shapiro, A. A. \& Stenby, E. H. 1996 On the nonequilibrium segregation state of a two-phase mixture in a porous column. Trans. Porous Med. 23 (1), 83-106.

Smith, E. H. 2012 The influence of small-scale heterogeneity on average relative permeability. In Reservoir Characterization II, p. 52. Academic.

Trevisan, L., Krishnamurthy, P. G. \& Meckel, T. A. $2017 a$ Impact of 3D capillary heterogeneity and bedform architecture at the sub-meter scale on $\mathrm{CO}_{2}$ saturation for buoyant flow in clastic aquifers. Intl J. Greenh. Gas Control 56, 237-249.

Trevisan, L., Illangasekare, T. H. \& Meckel, T. A. $2017 b$ Modelling plume behavior through a heterogeneous sand pack using a commercial invasion percolation model. Geomech. Geophys. Geo-Energy Geo-Resour. 3 (3), 327-337.

Wei, N., Gill, M., Crandall, D., Mcintyre, D., Wang, Y., Li, K. B. X. \& Bromhal, G. $2014 \mathrm{CO}_{2}$ flooding properties of liujiagou sandstone: influence of sub-core scale structure heterogeneity. Greenh. Gas. Sci. Technol. 4 (3), 400-418.

Wheaton, R. J. 1991 Treatment of variations of composition with depth in gas-condensate reservoirs (includes associated papers 23549 and 24109). SPE Res. Engng 6 (02), 239-244.

Yortsos, Y. C., SATIK, C., BACRI, J.-C. \& SAlin, D. 1993 Large-scale percolation theory of drainage. Trans. Porous Med. 10 (2), 171-195. 